\title{
On the new fractional hybrid boundary value problems with three-point integral hybrid conditions
}

\author{
D. Baleanu ${ }^{1,4}$, S. Etemad ${ }^{2,3}$, S. Pourrazi ${ }^{2,3}$ and Sh. Rezapour ${ }^{2,4^{*}}$
}

\author{
"Correspondence: \\ rezapourshahram@yahoo.ca \\ 2Department of Mathematics, \\ Azarbaijan Shahid Madani \\ University, Tabriz, Iran \\ ${ }^{4}$ Department of Medical Research, \\ China Medical University Hospital, \\ China Medical University, Taichung, \\ Taiwan \\ Full list of author information is \\ available at the end of the article
}

\begin{abstract}
We investigate some new class of hybrid type fractional differential equations and inclusions via some nonlocal three-point boundary value conditions. Also, we provide some examples to illustrate our results.
\end{abstract}

MSC: $34 \mathrm{~A} 08 ; 34 \mathrm{~A} 12$

Keywords: Boundary value problem; Dhage's fixed point; Fractional hybrid differential equation and inclusion

\section{Introduction}

In the last two decades, the notion of initial and boundary value problems of fractional order has received considerable attention due to its extensive developments and numerous applications connected with natural phenomena in the real world. Some examples of these applications that have appeared in many applied aspects of the basic sciences and the advanced engineering are: chemistry, fractal theory, optics, control theory, biology, biophysics, economics, etc. For this importance, one can find many papers published in this field. From the mathematical viewpoint, see e.g. [1, 3, 6, 9-22, 28, 29, 33-35] and the references therein.

More recently, new developments and research in fractional hybrid differential equations and inclusions have been made. First time, in 2010 Dhage and Lakshmikantham introduced hybrid differential equations and proved some basic results on this kind of differential equations [26].

Next, Zhao et al. [40] generalized Dhage's work to fractional order and studied theory of fractional differential equations of hybrid type. Since then, many researchers have been interested in working on the existence topics for different fractional hybrid differential equations and inclusions. For instance, Sun et al. [39] defined the two-point boundary value problem of fractional hybrid differential equation

$$
\begin{aligned}
& \mathcal{D}_{0}^{q}\left[\frac{z(\tau)}{f(\tau, z(\tau))}\right]+g(\tau, z(\tau))=0, \quad \tau \in[0,1], \\
& z(0)=z(1)=0,
\end{aligned}
$$

\section{Springer}

(c) The Author(s) 2019. This article is distributed under the terms of the Creative Commons Attribution 4.0 International License (http://creativecommons.org/licenses/by/4.0/), which permits unrestricted use, distribution, and reproduction in any medium, provided you give appropriate credit to the original author(s) and the source, provide a link to the Creative Commons license, and indicate if changes were made. 
where $q \in(1,2]$ and $\mathcal{D}_{0}^{\alpha}$ denotes the fractional order derivative operator defined by Riemann-Liouville. Authors proved existence theorems by applying Dhage's fixed point theorem in Banach algebra under the mixed Caratheodory and Lipschitz conditions.

In 2014, Ahmad, Ntouyas, and Alsaedi investigated the results on the existence of coupled solutions for the new coupled system of the fractional differential equations of hybrid type

$$
\begin{aligned}
& { }^{c} \mathcal{D}_{0}^{\delta}\left(\frac{z_{1}(\tau)}{f_{1}\left(\tau, z_{1}(\tau), z_{2}(\tau)\right)}\right)=k_{1}\left(\tau, z_{1}(\tau), z_{2}(\tau)\right), \\
& { }^{c} \mathcal{D}_{0}^{\omega}\left(\frac{z_{2}(\tau)}{f_{2}\left(\tau, z_{1}(\tau), z_{2}(\tau)\right)}\right)=k_{2}\left(\tau, z_{1}(\tau), z_{2}(\tau)\right), \quad \tau \in[0,1],
\end{aligned}
$$

with Dirichlet boundary conditions

$$
z_{1}(0)=z_{1}(1)=0, \quad z_{2}(0)=z_{2}(1)=0,
$$

where ${ }^{c} \mathcal{D}_{0}^{\gamma}$ is the derivative operator of the fractional order $\gamma \in\{\delta, \omega\} \subset(1,2]$ of Caputo type. Authors described the uniqueness theorems for above hybrid problem by the aid of the Banach contraction principle and showed the existence results by means of the nonlinear alternative of Leray-Schauder type [5].

In 2016, Baleanu et al. [13] discussed the existence theorems for the fractional hybrid differential inclusion

$$
{ }^{c} \mathcal{D}_{0}^{p^{*}}\left(\frac{z(\tau)}{f\left(\tau, z(\tau), \mathcal{I}_{0}^{\alpha_{1}} z(\tau), \ldots, \mathcal{I}_{0}^{\alpha_{n}} z(\tau)\right)}\right) \in \mathcal{H}\left(\tau, z(\tau), \mathcal{I}_{0}^{\beta_{1}} z(\tau), \ldots, \mathcal{I}_{0}^{\beta_{k}} z(\tau)\right), \quad \tau \in[0,1]
$$

with boundary value conditions $z(0)=z_{0}^{*}$ and $z(1)=z_{1}^{*}$, where $p^{*} \in(1,2]$. Also ${ }^{c} \mathcal{D}_{0}^{p^{*}}$ and $\mathcal{I}_{0}^{\gamma}$ denote the Caputo derivative operator of the fractional order $p^{*}$ and the RiemannLiouville integral operator of the fractional order $\gamma \in\left\{\alpha_{i}, \beta_{j}\right\} \subset(0, \infty)$ for $i=1, \ldots, n$ and $j=1, \ldots, k$, respectively. The existence theorem for the above hybrid inclusion problem is proved by using Dhage's fixed point theorem for multi-valued maps.

Also, we can find some other same works published on the fractional hybrid boundary value problem in $[2,4,7,14,27,31,37]$ and the references therein.

Motivated by the above-mentioned works, we investigate a new class of fractional hybrid differential equations and inclusions. Indeed, we first investigate the fractional hybrid differential equation

$$
{ }^{c} \mathcal{D}_{0}^{p^{*}}\left(\frac{z(\tau)}{\rho(\tau, z(\tau))}\right)=\kappa(\tau, z(\tau)), \quad \tau \in[0,1]
$$

via three-point integral hybrid conditions

$$
\begin{aligned}
& z(0)=0, \\
& \left.\left(\frac{z(\tau)}{\rho(\tau, z(\tau))}\right)\right|_{\tau=0}+\left.\mathcal{J}_{0}^{q^{*}}\left(\frac{z(\tau)}{\rho(\tau, z(\tau))}\right)\right|_{\tau=\eta}=0, \\
& \left.\left(\frac{z(\tau)}{\rho(\tau, z(\tau))}\right)\right|_{\tau=0}+\left.\mathcal{J}_{0}^{q^{*}}\left(\frac{z(\tau)}{\rho(\tau, z(\tau))}\right)\right|_{\tau=1}=0,
\end{aligned}
$$


where $p^{*} \in(2,3], q^{*}>0$, and $\eta \in(0,1)$. Also, ${ }^{c} \mathcal{D}_{0}^{p^{*}}$ and $\mathcal{J}_{0}^{q^{*}}$ are the Caputo derivative operator of the fractional order $p^{*}$ and the Riemann-Liouville integral operator of the fractional order $q^{*}$, respectively. The function $\kappa:[0,1] \times \mathbb{R} \rightarrow \mathbb{R}$ is continuous and $\rho \in C([0,1] \times \mathbb{R}, \mathbb{R} \backslash\{0\})$. We are devoted to establishing some basic theorems on the existence of solutions by means of Dhage's nonlinear alternative of Schaefer type for this boundary value problem.

In the next step, we deal with the fractional hybrid differential inclusion

$$
{ }^{c} \mathcal{D}_{0}^{p^{*}}\left(\frac{z(\tau)}{\rho(\tau, z(\tau))}\right) \in \mathcal{H}(\tau, z(\tau)), \quad \tau \in[0,1]
$$

with three-point integral hybrid conditions (2), where $\mathcal{H}:[0,1] \times \mathbb{R} \rightarrow \mathcal{P}(\mathbb{R})$ is a multifunction via some other properties. By virtue of Dhage's fixed point theorem for multivalued maps, the existence and uniqueness results were obtained.

It is noted that the fractional differential equation and inclusion of hybrid type presented in this paper are elementary and new in the sense that the boundary value conditions are as nonlocal three-point integral hybrid conditions. Also, this hybrid boundary value problem is general and it involves many fractional dynamical systems as special cases. It is sufficient to set $\rho(\tau, z(\tau))=1$ as a constant function. In this case, the hybrid boundary value problem (1)-(2) will reduce to an ordinary fractional boundary value problem as follows:

$$
\begin{aligned}
& { }^{c} \mathcal{D}_{0}^{p^{*}} z(\tau)=\kappa(\tau, z(\tau)), \quad \tau \in[0,1], \\
& z(0)=0, \quad z(0)+\mathcal{J}_{0}^{q^{*}} z(\eta)=0, \quad z(0)+\mathcal{J}_{0}^{q^{*}} z(1)=0,
\end{aligned}
$$

where $p^{*} \in(2,3], q^{*}>0$, and $\eta \in(0,1)$.

The structure of the paper is as follows. In Sect. 2, we provide some basic definitions and theorems which are needed in the sequel. Section 3 specifies the hybrid boundary value problems that we shall investigate. The main existence theorems are given in this section. Finally, some illustrative examples are presented in the last section.

\section{Preliminaries}

The present section specifies some important and applied definitions and theorems on fractional calculus and multifunctions that we shall recall.

Let us assume that $p^{*}>0$ is a real number with $n-1<p^{*}<n, n=\left[p^{*}\right]+1$. The RiemannLiouville integral of the function $z \in C\left(\left[t_{1}, t_{2}\right], \mathbb{R}\right)$ of the fractional order is given by

$$
\mathcal{J}_{0}^{p^{*}} z(\tau)=\frac{1}{\Gamma\left(p^{*}\right)} \int_{0}^{\tau} \frac{z(m)}{(\tau-m)^{1-p^{*}}} \mathrm{~d} m, \quad \tau>0
$$

whenever the integral exists [36, 38].

The Caputo derivative of the function $z \in C^{(n)}\left(\left[t_{1}, t_{2}\right], \mathbb{R}\right)$ of the fractional order is defined by

$$
{ }^{c} \mathcal{D}_{0}^{p^{*}} z(\tau)=\frac{1}{\Gamma\left(n-p^{*}\right)} \int_{0}^{\tau}(\tau-m)^{n-p^{*}-1} z^{(n)}(m) \mathrm{d} m
$$

$[36,38]$. Note that the general solution for ${ }^{c} \mathcal{D}_{0}^{p^{*}} z(\tau)=0$ is obtained by relation $z(\tau)=c_{0}+$ $c_{1} \tau+c_{2} \tau^{2}+\cdots+c_{n-1} \tau^{n-1}$, where $c_{0}, \ldots, c_{n-1}$ denote the real numbers and $n=\left[p^{*}\right]+1[32]$. 
Also, for every positive real number $T^{*}$ and every continuous function $z$ on $\left[0, T^{*}\right]$, we have

$$
\left(\mathcal{J}_{0}^{p^{*} c} \mathcal{D}_{0}^{p^{*}}\right) z(\tau)=z(\tau)+c_{0}+c_{1} \tau+c_{2} \tau^{2}+\cdots+c_{n-1} \tau^{n-1}
$$

where $c_{0}, \ldots, c_{n-1}$ are constants belonging to $\mathbb{R}$ and $n=\left[p^{*}\right]+1$ [32].

Let $\mathcal{X}$ be a normed space with relevant norm function $\|\cdot\|_{\mathcal{X}}$. We denote by notations $\mathcal{P}(\mathcal{X}), \mathcal{P}_{c l}(\mathcal{X}), \mathcal{P}_{b}(\mathcal{X}), \mathcal{P}_{c p}(\mathcal{X})$, and $\mathcal{P}_{c p, c v}(\mathcal{X})$ all subsets of the space $\mathcal{X}$, all nonempty closed subsets of the space $\mathcal{X}$, all nonempty bounded subsets of the space $\mathcal{X}$, all nonempty compact subsets of the space $\mathcal{X}$, and all nonempty compact convex subsets of the space $\mathcal{X}$, respectively.

An element $z^{*} \in \mathcal{X}$ is called a fixed point of a multi-valued function $\mathcal{H}: \mathcal{X} \rightarrow \mathcal{P}(\mathcal{X})$ if $z^{*} \in \mathcal{H}\left(z^{*}\right)$. All fixed points of the multifunction $\mathcal{H}$ is a set that will be denoted by notation Fix $(\mathcal{H})$ [23]. A multifunction $\mathcal{H}$ is called convex-valued if the set $\mathcal{H}(z)$ is convex for each element $z \in \mathcal{X}$. Also, we say that the multifunction $\mathcal{H}$ is an upper semi-continuous (u.s.c.) on the space $\mathcal{X}$ if, for every arbitrary element $z^{*} \in \mathcal{X}$, the set $\mathcal{H}\left(z^{*}\right)$ belongs to $\mathcal{P}_{c l}(\mathcal{X})$ and also, if for every arbitrary open set $V$ of $\mathcal{X}$ containing $\mathcal{H}\left(z^{*}\right)$, there exists an open neighborhood $\mathcal{N}_{0}^{*}$ of $z^{*}$ provided that $\mathcal{H}\left(\mathcal{N}_{0}^{*}\right) \subseteq V$ [23].

Now, let us assume that the pair $(\mathcal{X}, d)$ is a metric space. The Pompeiu-Hausdorff metric $P H_{d}: \mathcal{P}(\mathcal{X}) \times \mathcal{P}(\mathcal{X}) \rightarrow \mathbb{R} \cup\{\infty\}$ is defined by

$$
P H_{d}\left(M^{*}, N^{*}\right)=\max \left\{\sup _{m^{*} \in M^{*}} d\left(m^{*}, N^{*}\right), \sup _{n^{*} \in N^{*}} d\left(M^{*}, n^{*}\right)\right\}
$$

where $d\left(M^{*}, n^{*}\right)=\inf _{m^{*} \in M^{*}} d\left(m^{*}, n^{*}\right)$ and $d\left(m^{*}, N^{*}\right)=\inf _{n^{*} \in N^{*}} d\left(m^{*}, n^{*}\right)$. A multi-valued function $\mathcal{H}: \mathcal{X} \rightarrow \mathcal{P}_{c l}(\mathcal{X})$ is called Lipschitzian with Lipschitz constant $k>0$ if

$$
P H_{d}\left(\mathcal{H}\left(z_{1}\right), \mathcal{H}\left(z_{2}\right)\right) \leq k d\left(z_{1}, z_{2}\right)
$$

for each $z_{1}, z_{2} \in \mathcal{X}$. A Lipschitz map $\mathcal{H}$ is called contraction if $k \in(0,1)$ [23].

For the multi-valued function $\mathcal{H}: \mathcal{X} \rightarrow \mathcal{P}_{c l}(\mathcal{Y})$, the graph $\mathcal{H}$ denoted by $\operatorname{Gr}(\mathcal{H})$ is the set $\operatorname{Gr}(\mathcal{H})=\left\{\left(z_{1}, z_{2}\right) \in \mathcal{X} \times \mathcal{Y}: w^{*} \in \mathcal{H}(z)\right\}[23]$. The graph $\operatorname{Gr}(\mathcal{H})$ of $\mathcal{H}$ is said to be a closed subset of the product space $\mathcal{X} \times \mathcal{Y}$ if, for every sequence $\left\{z_{n}\right\}_{n \geq 1}$ in $\mathcal{X}$ and $\left\{w_{n}\right\}_{n \geq 1}$ in $\mathcal{Y}$, $z_{n} \rightarrow z_{0}, w_{n} \rightarrow w_{0}$, and $w_{n} \in \mathcal{H}\left(z_{n}\right)$, then $w_{0} \in \mathcal{H}\left(z_{0}\right)$ whenever $n \rightarrow \infty$ [8]. In this case, one can say that the multifunction $\mathcal{H}$ has a closed graph. We say that a multifunction $\mathcal{H}$ is a completely continuous operator if the set $\mathcal{H}(\mathcal{B})$ is relatively compact for each $\mathcal{B} \in \mathcal{P}_{b}(\mathcal{X})$. If the multifunction $\mathcal{H}: \mathcal{X} \rightarrow \mathcal{P}_{c l}(\mathcal{Y})$ is an upper semi-continuous, then $\operatorname{Gr}(\mathcal{H})$ is a subset of the product space $\mathcal{X} \times \mathcal{Y}$ with closedness property. Conversely, if $\mathcal{H}$ is a completely continuous multifunction and has a closed graph, then $\mathcal{H}$ is an operator with upper semicontinuity property ([23], Proposition 2.1).

A multifunction $\mathcal{H}:[0,1] \rightarrow \mathcal{P}_{c l}(\mathbb{R})$ is called measurable if, for all real constants $\omega$, the function $\tau \mapsto d(\omega, \mathcal{H}(\tau))=\inf \{|\omega-\nu|: \nu \in \mathcal{H}(\tau)\}$ is measurable [8, 23].

We say that $\mathcal{H}:[0,1] \times \mathbb{R} \rightarrow \mathcal{P}(\mathbb{R})$ is a Caratheodory multifunction if $\tau \mapsto \mathcal{H}(\tau, z)$ is a measurable mapping for every element $z \in \mathbb{R}$ and $z \mapsto \mathcal{H}(\tau, z)$ is an upper semi-continuous mapping for almost all $\tau \in[0,1][8,23]$. Also, a Caratheodory multifunction $\mathcal{H}:[0,1] \times$ $\mathbb{R} \rightarrow \mathcal{P}(\mathbb{R})$ is said to be $\mathcal{L}^{1}$-Caratheodory if, for every constant $\mu>0$, there exists a function 
$\phi_{\mu} \in \mathcal{L}^{1}\left([0,1], \mathbb{R}^{+}\right)$such that

$$
\|\mathcal{H}(\tau, z)\|=\sup _{\tau \in[0,1]}\{|s|: s \in \mathcal{H}(\tau, z)\} \leq \phi_{\mu}(\tau)
$$

for all $|z| \leq \mu$ and for almost all $\tau \in[0,1][8,23]$. The set of selections of a multifunction $\mathcal{H}$ at point $z \in C([0,1], \mathbb{R})$ is defined by

$$
S_{\mathcal{H}, z}:=\left\{v \in \mathcal{L}^{1}([0,1], \mathbb{R}): v(\tau) \in \mathcal{H}(\tau, z(\tau))\right\}
$$

for almost all $\tau \in[0,1]$. We assume that $\mathcal{H}$ is an arbitrary multifunction. Then the authors in [23] showed that $S_{\mathcal{H}, z} \neq \emptyset$ for all $z \in C([0,1], \mathcal{X})$ if $\operatorname{dim} \mathcal{X}<\infty$.

Finally, we will use the following three theorems to obtain the main results.

Theorem 2.1 ([25] (Dhage's nonlinear alternative of Schaefer type)) Let $\mathcal{X}$ be a Banach algebra. For some positive number $r \in \mathbb{R}$, consider an open ball $V_{\epsilon}(0)$ and a closed ball $\bar{V}_{\epsilon}(0)$ in $\mathcal{X}$. Suppose that two operators $A_{1}: \mathcal{X} \rightarrow \mathcal{X}$ and $A_{2}: \bar{V}_{\epsilon}(0) \rightarrow \mathcal{X}$ satisfy the following conditions:

(i) $A_{1}$ is an operator including the Lipschitzian property with a Lipschitz constant $k^{*}$;

(ii) $A_{2}$ has the complete continuity property;

(iii) $k^{*} M_{0}^{*}<1$, so that $M_{0}^{*}=\left\|A_{2}\left(\bar{V}_{\epsilon}(0)\right)\right\|_{\mathcal{X}}=\sup \left\{\left\|A_{2} z\right\|_{\mathcal{X}}: z \in \bar{V}_{\epsilon}(0)\right\}$.

Then either

(a) there is a solution in $\bar{V}_{\epsilon}(0)$ for the operator equation $A_{1} z A_{2} z=z$, or

(b) there is an element $v^{*} \in \mathcal{X}$ with $\left\|v^{*}\right\|_{\mathcal{X}}=r$ such that $\lambda A_{1} v^{*} A_{2} v^{*}=v^{*}$ for some $\lambda \in(0,1)$.

Theorem 2.2 ([30]) Suppose that $\mathcal{X}$ is a separable Banach space, $\mathcal{H}:[0,1] \times \mathcal{X} \rightarrow$ $\mathcal{P}_{c p, c v}(\mathcal{X})$ is an $\mathcal{L}^{1}$-Caratheodory multifunction and $\Theta: \mathcal{L}^{1}([0,1], \mathcal{X}) \rightarrow C([0,1], \mathcal{X})$ is a linear continuous mapping. Then $\Theta \circ S_{\mathcal{H}}: C([0,1], \mathcal{X}) \rightarrow \mathcal{P}_{c p, c v}(C([0,1], \mathcal{X}))$ is an operator in the product space $C([0,1], \mathcal{X}) \times C([0,1], \mathcal{X})$ with action $z \mapsto\left(\Theta \circ S_{\mathcal{H}}\right)(z)=\Theta\left(S_{\mathcal{H}, z}\right)$ having the closed graph property.

Theorem 2.3 ([24]) Suppose that $X$ is a Banach algebra. Suppose that there exist a singlevalued map $A_{1}: \mathcal{X} \rightarrow \mathcal{X}$ and a multi-valued map $A_{2}: \mathcal{X} \rightarrow \mathcal{P}_{c p, c v}(\mathcal{X})$ such that

(i) $A_{1}$ is an operator including the Lipschitzian property with a Lipschitz constant $k^{*}$;

(ii) $A_{2}$ is an operator including the upper semi-continuity and compactness property;

(iii) $2 k^{*} M_{0}^{*}<1$ so that $M_{0}^{*}=\left\|A_{2}(\mathcal{X})\right\|$.

Then either

(a) there is a solution in $\mathcal{X}$ for the operator inclusion $z \in A_{1} z A_{2} z$, or

(b) the set $\Sigma^{*}=\left\{v^{*} \in \mathcal{X} \mid \mu v^{*} \in A_{1} v^{*} A_{2} v^{*}, \mu>1\right\}$ is not bounded.

\section{Existence results}

Suppose that $\mathcal{X}=C([0,1], \mathbb{R})$ is the space of all continuous functions on $[0,1]$. For each $z \in \mathcal{X}$, set $\|z\|_{\mathcal{X}}=\sup \{|z(\tau)|: \tau \in[0,1]\}$. Then $\mathcal{X}$ is a Banach space endowed with this norm. Now, we state the following basic lemma. 
Lemma 3.1 Assume that the continuous real-valued function $\varphi$ is defined on $[0,1]$. The solution function $z_{0}^{*}$ for the fractional hybrid differential equation is given by

$$
{ }^{c} \mathcal{D}_{0}^{p^{*}}\left(\frac{z(\tau)}{\rho(\tau, z(\tau))}\right)=\varphi(\tau), \quad \tau \in[0,1], p^{*} \in(2,3]
$$

with three-point integral hybrid conditions

$$
\begin{aligned}
& z(0)=0, \\
& \left.\left(\frac{z(\tau)}{\rho(\tau, z(\tau))}\right)\right|_{\tau=0}+\left.\mathcal{J}_{0}^{q^{*}}\left(\frac{z(\tau)}{\rho(\tau, z(\tau))}\right)\right|_{\tau=\eta}=0, \\
& \left.\left(\frac{z(\tau)}{\rho(\tau, z(\tau))}\right)\right|_{\tau=0}+\left.\mathcal{J}_{0}^{q^{*}}\left(\frac{z(\tau)}{\rho(\tau, z(\tau))}\right)\right|_{\tau=1}=0
\end{aligned}
$$

if and only if the solution function $z_{0}^{*}$ for an integral equation of the fractional order is given by

$$
\begin{aligned}
z(\tau)= & \rho(\tau, z(\tau))\left[\int_{0}^{\tau} \frac{(\tau-m)^{p^{*}-1}}{\Gamma\left(p^{*}\right)} \varphi(m) \mathrm{d} m-\frac{\tau \Gamma\left(q^{*}+2\right)}{\eta^{q^{*}+1}} \int_{0}^{\eta} \frac{(\eta-m)^{p^{*}+q^{*}-1}}{\Gamma\left(p^{*}+q^{*}\right)} \varphi(m) \mathrm{d} m\right. \\
& +\frac{\tau \Gamma\left(q^{*}+2\right)\left(\eta-\eta^{q^{*}+2}\right)}{\left(\eta^{q^{*}+2}-\eta^{q^{*}+1}\right)} \int_{0}^{1} \frac{(1-m)^{p^{*}+q^{*}-1}}{\Gamma\left(p^{*}+q^{*}\right)} \varphi(m) \mathrm{d} m \\
& +\frac{\tau^{2} \Gamma\left(q^{*}+3\right)}{(\eta-1)} \int_{0}^{1} \frac{(1-m)^{p^{*}+q^{*}-1}}{\Gamma\left(p^{*}+q^{*}\right)} \varphi(m) \mathrm{d} m \\
& \left.-\frac{\tau^{2} \Gamma\left(q^{*}+3\right)}{\left(\eta^{q^{*}+2}-\eta^{q^{*}+1}\right)} \int_{0}^{\eta} \frac{(\eta-m)^{p^{*}+q^{*}-1}}{\Gamma\left(p^{*}+q^{*}\right)} \varphi(m) \mathrm{d} m\right]
\end{aligned}
$$

Proof Let $z_{0}^{*}$ be a solution function for the fractional hybrid differential equation (4). Then there exist constants $c_{0}, c_{1}, c_{2} \in \mathbb{R}$ provided that

$$
z_{0}^{*}(\tau)=\rho\left(\tau, z_{0}^{*}(\tau)\right)\left[\int_{0}^{\tau} \frac{(\tau-m)^{p^{*}-1}}{\Gamma\left(p^{*}\right)} \varphi(m) \mathrm{d} m+c_{0}+c_{1} \tau+c_{2} \tau^{2}\right] .
$$

Also, for $q^{*}>0$, we have

$$
\begin{aligned}
\mathcal{J}_{0}^{q^{*}}\left(\frac{z_{0}^{*}(\tau)}{\rho\left(\tau, z_{0}^{*}(\tau)\right)}\right)= & \int_{0}^{\tau} \frac{(\tau-m)^{p^{*}+q^{*}-1}}{\Gamma\left(p^{*}+q^{*}\right)} \varphi(m) \mathrm{d} m \\
& +c_{0} \frac{\tau^{q^{*}}}{\Gamma\left(q^{*}+1\right)}+c_{1} \frac{\tau^{q^{*}+1}}{\Gamma\left(q^{*}+2\right)}+c_{2} \frac{\tau^{q^{*}+2}}{\Gamma\left(q^{*}+3\right)}
\end{aligned}
$$

By using the initial condition $z_{0}^{*}(0)=0$, we get $c_{0}=0$ and by the rest of the boundary value conditions (5), we have

$$
\begin{aligned}
c_{1}= & -\frac{\Gamma\left(q^{*}+2\right)}{\eta^{q^{*}+1}} \int_{0}^{\eta} \frac{(\eta-m)^{p^{*}+q^{*}-1}}{\Gamma\left(p^{*}+q^{*}\right)} \varphi(m) \mathrm{d} m \\
& +\frac{\Gamma\left(q^{*}+2\right)\left(\eta-\eta^{q^{*}+2}\right)}{\left(\eta^{q^{*}+2}-\eta^{q^{*}+1}\right)} \int_{0}^{1} \frac{(1-m)^{p^{*}+q^{*}-1}}{\Gamma\left(p^{*}+q^{*}\right)} \varphi(m) \mathrm{d} m
\end{aligned}
$$


and

$$
\begin{aligned}
c_{2}= & \frac{\eta^{q^{*}+1} \Gamma\left(q^{*}+3\right)}{\left(\eta^{q^{*}+2}-\eta^{q^{*}+1}\right)} \int_{0}^{1} \frac{(1-m)^{p^{*}+q^{*}-1}}{\Gamma\left(p^{*}+q^{*}\right)} \varphi(m) \mathrm{d} m \\
& -\frac{\Gamma\left(q^{*}+3\right)}{\left(\eta^{q^{*}+2}-\eta^{q^{*}+1}\right)} \int_{0}^{\eta} \frac{(\eta-m)^{p^{*}+q^{*}-1}}{\Gamma\left(p^{*}+q^{*}\right)} \varphi(m) \mathrm{d} m .
\end{aligned}
$$

Substituting the values $c_{i}(i=0,1,2)$ in $(7)$, we get $(6)$ showing that $z_{0}^{*}$ is a solution function for the fractional integral equation (6). Conversely, one can easily see that $z_{0}^{*}$ is a solution function for the hybrid boundary value problem of fractional order (4)-(5) whenever $z_{0}^{*}$ is a solution function for the fractional integral equation (6).

Theorem 3.2 Assume that $\rho \in C([0,1] \times \mathbb{R}, \mathbb{R} \backslash\{0\})$ and $\kappa \in C([0,1] \times \mathbb{R}, \mathbb{R})$. Also, we have the following assumptions:

(H1) There is a bounded mapping $L:[0,1] \rightarrow \mathbb{R}^{+}$such that, for all $z, w \in \mathbb{R}$, we have

$$
|\rho(\tau, z)-\rho(\tau, w)| \leq L(\tau)|z(\tau)-w(\tau)|
$$

(H2) There are a continuous nondecreasing mapping $\zeta:[0, \infty) \rightarrow(0, \infty)$ and a continuous function $\sigma:[0,1] \rightarrow \mathbb{R}^{+}$provided that, for $\tau \in[0,1]$ and for all $z \in \mathbb{R}$, we have

$$
|\kappa(\tau, z)| \leq \sigma(\tau) \zeta(\|z\|)
$$

(H3) There is a positive number $\epsilon \in \mathbb{R}$ such that

$$
\epsilon>\frac{F^{*} \Delta M^{*} \zeta(\|z\|)}{1-L^{*} \Delta M^{*} \zeta(\|z\|)}
$$

where $F^{*}=\sup _{\tau \in[0,1]}|\rho(\tau, 0)|, M^{*}=\sup _{\tau \in[0,1]}|\sigma(\tau)|, L^{*}=\sup _{\tau \in[0,1]}|L(\tau)|$, and

$$
\begin{aligned}
\Delta= & \frac{1}{\Gamma\left(p^{*}+1\right)}+\frac{\Gamma\left(q^{*}+2\right) \eta^{p^{*}-1}}{\Gamma\left(p^{*}+q^{*}+1\right)}+\frac{\Gamma\left(q^{*}+2\right)\left|\eta-\eta^{q^{*}+2}\right|}{\left(\eta^{q^{*}+2}-\eta^{q^{*}+1}\right) \Gamma\left(p^{*}+q^{*}+1\right)} \\
& +\frac{\Gamma\left(q^{*}+3\right)}{(\eta-1) \Gamma\left(p^{*}+q^{*}+1\right)}+\frac{\Gamma\left(q^{*}+3\right) \eta^{p^{*}+q^{*}}}{\left(\eta^{q^{*}+2}-\eta^{q^{*}+1}\right) \Gamma\left(p^{*}+q^{*}+1\right)} .
\end{aligned}
$$

If $L^{*} \Delta M^{*} \zeta(\|z\|)<1$, then the fractional hybrid BVP (1)-(2) has at least one solution on an interval $[0,1]$.

Proof Consider the closed ball $\bar{V}_{\epsilon}(0):=\left\{z \in \mathcal{X}:\|z\|_{\mathcal{X}} \leq \epsilon\right\}$ in a Banach space $\mathcal{X}$, where $r$ satisfies inequality (8). By Lemma 3.1 and by the fractional integral equation (6), we define two operators $A_{1}, A_{2}: \bar{V}_{\epsilon}(0) \rightarrow \mathcal{X}$ by

$$
\left(A_{1} z\right)(\tau)=\rho(\tau, z(\tau))
$$


and

$$
\begin{aligned}
\left(A_{2} z\right)(\tau)= & \int_{0}^{\tau} \frac{(\tau-m)^{p^{*}-1}}{\Gamma\left(p^{*}\right)} \kappa(m, z(m)) \mathrm{d} m \\
& -\frac{\tau \Gamma\left(q^{*}+2\right)}{\eta^{q^{*}+1}} \int_{0}^{\eta} \frac{(\eta-m)^{p^{*}+q^{*}-1}}{\Gamma\left(p^{*}+q^{*}\right)} \kappa(m, z(m)) \mathrm{d} m \\
& +\frac{\tau \Gamma\left(q^{*}+2\right)\left(\eta-\eta^{q^{*}+2}\right)}{\left(\eta^{q^{*}+2}-\eta^{q^{*}+1}\right)} \int_{0}^{1} \frac{(1-m)^{p^{*}+q^{*}-1}}{\Gamma\left(p^{*}+q^{*}\right)} \kappa(m, z(m)) \mathrm{d} m \\
& +\frac{\tau^{2} \Gamma\left(q^{*}+3\right)}{(\eta-1)} \int_{0}^{1} \frac{(1-m)^{p^{*}+q^{*}-1}}{\Gamma\left(p^{*}+q^{*}\right)} \kappa(m, z(m)) \mathrm{d} m \\
& -\frac{\tau^{2} \Gamma\left(q^{*}+3\right)}{\left(\eta^{q^{*}+2}-\eta^{q^{*}+1}\right)} \int_{0}^{\eta} \frac{(\eta-m)^{p^{*}+q^{*}-1}}{\Gamma\left(p^{*}+q^{*}\right)} \kappa(m, z(m)) \mathrm{d} m .
\end{aligned}
$$

Then it is evident that the function $z \in \mathcal{X}$ as a solution function for the fractional hybrid BVP (1)-(2) satisfies the operator equation $A_{1} z A_{2} z=z$. To see this, we prove that there exists such a solution based on the assumptions of Theorem 2.1.

In the first step, it is proved that the operator $A_{1}$ is Lipschitz on a normed space $X$ with Lipschitz constant $L^{*}=\sup _{\tau \in[0,1]}|L(\tau)|$. For each $z, w \in \mathcal{X}$ and by (H1), we get

$$
\begin{aligned}
\left|\left(A_{1} z\right)(\tau)-\left(A_{1} w\right)(\tau)\right| & =|\rho(\tau, z(\tau))-\rho(\tau, w(\tau))| \\
& \leq L(\tau)|z(\tau)-w(\tau)|
\end{aligned}
$$

for each $z, w \in \bar{V}_{\epsilon}(0)$. Now, we take the supremum over [0,1],

$$
\left\|A_{1} z-A_{1} w\right\|_{\mathcal{X}} \leq L^{*}\|z-w\|_{\mathcal{X}}, \quad z, w \in \bar{V}_{\epsilon}(0),
$$

showing that the operator $A_{1}$ is Lipschitzian on $\bar{V}_{\epsilon}(0)$ including Lipschitz constant $L^{*}$.

Now, we prove that the operator $A_{2}$ is completely continuous on $\bar{V}_{\epsilon}(0)$. For this, we first prove the continuity of the operator $A_{2}$ on $\bar{V}_{\epsilon}(0)$. Suppose that $\left\{z_{n}\right\}$ is a convergent sequence in an open ball $\bar{V}_{\epsilon}(0)$ such that $z_{n} \rightarrow z$ as $n \rightarrow \infty$, where $z$ is an element belonging to $\bar{V}_{\epsilon}(0)$. Since $\kappa$ is continuous on $[0,1] \times \mathbb{R}$, thus $\lim _{n \rightarrow \infty} \kappa\left(\tau, z_{n}(\tau)\right)=\kappa(\tau, z(\tau))$. Then, by Lebesgue's dominated convergence theorem, we deduce that

$$
\begin{aligned}
\lim _{n \rightarrow \infty}\left(A_{2} z_{n}\right)(\tau)= & \int_{0}^{\tau} \frac{(\tau-m)^{p^{*}-1}}{\Gamma\left(p^{*}\right)} \lim _{n \rightarrow \infty} \kappa\left(m, z_{n}(m)\right) \mathrm{d} m \\
& -\frac{\tau \Gamma\left(q^{*}+2\right)}{\eta^{q^{*}+1}} \int_{0}^{\eta} \frac{(\eta-m)^{p^{*}+q^{*}-1}}{\Gamma\left(p^{*}+q^{*}\right)} \lim _{n \rightarrow \infty} \kappa\left(m, z_{n}(m)\right) \mathrm{d} m \\
& +\frac{\tau \Gamma\left(q^{*}+2\right)\left(\eta-\eta^{q^{*}+2}\right)}{\left(\eta^{q^{*}+2}-\eta^{q^{*}+1}\right)} \int_{0}^{1} \frac{(1-m)^{p^{*}+q^{*}-1}}{\Gamma\left(p^{*}+q^{*}\right)} \lim _{n \rightarrow \infty} \kappa\left(m, z_{n}(m)\right) \mathrm{d} m \\
& +\frac{\tau^{2} \Gamma\left(q^{*}+3\right)}{(\eta-1)} \int_{0}^{1} \frac{(1-m)^{p^{*}+q^{*}-1}}{\Gamma\left(p^{*}+q^{*}\right)} \lim _{n \rightarrow \infty} \kappa\left(m, z_{n}(m)\right) \mathrm{d} m \\
& -\frac{\tau^{2} \Gamma\left(q^{*}+3\right)}{\left(\eta^{q^{*}+2}-\eta^{q^{*}+1}\right)} \int_{0}^{\eta} \frac{(\eta-m)^{p^{*}+q^{*}-1}}{\Gamma\left(p^{*}+q^{*}\right)} \lim _{n \rightarrow \infty} \kappa\left(m, z_{n}(m)\right) \mathrm{d} m \\
= & \int_{0}^{\tau} \frac{(\tau-m)^{p^{*}-1}}{\Gamma\left(p^{*}\right)} \kappa(m, z(m)) \mathrm{d} m
\end{aligned}
$$




$$
\begin{aligned}
& -\frac{\tau \Gamma\left(q^{*}+2\right)}{\eta^{q^{*}+1}} \int_{0}^{\eta} \frac{(\eta-m)^{p^{*}+q^{*}-1}}{\Gamma\left(p^{*}+q^{*}\right)} \kappa(m, z(m)) \mathrm{d} m \\
& +\frac{\tau \Gamma\left(q^{*}+2\right)\left(\eta-\eta^{q^{*}+2}\right)}{\left(\eta^{q^{*}+2}-\eta^{q^{*}+1}\right)} \int_{0}^{1} \frac{(1-m)^{p^{*}+q^{*}-1}}{\Gamma\left(p^{*}+q^{*}\right)} \kappa(m, z(m)) \mathrm{d} m \\
& +\frac{\tau^{2} \Gamma\left(q^{*}+3\right)}{(\eta-1)} \int_{0}^{1} \frac{(1-m)^{p^{*}+q^{*}-1}}{\Gamma\left(p^{*}+q^{*}\right)} \kappa(m, z(m)) \mathrm{d} m \\
& -\frac{\tau^{2} \Gamma\left(q^{*}+3\right)}{\left(\eta^{q^{*}+2}-\eta^{q^{*}+1}\right)} \int_{0}^{\eta} \frac{(\eta-m)^{p^{*}+q^{*}-1}}{\Gamma\left(p^{*}+q^{*}\right)} \kappa(m, z(m)) \mathrm{d} m \\
& =\left(A_{2} z\right)(\tau)
\end{aligned}
$$

for all $\tau \in[0,1]$. Therefore $A_{2} z_{n} \rightarrow A_{2} z$, which shows that $A_{2}$ is continuous on $\bar{V}_{\epsilon}(0)$.

In the next step, the uniform boundedness of operator $A_{2}$ on $\bar{V}_{\epsilon}(0)$ is proved. By assumption (H2) and for all $\tau \in[0,1]$, one can write

$$
\begin{aligned}
\left|\left(A_{2} z\right)(\tau)\right|= & \int_{0}^{\tau} \frac{(\tau-m)^{p^{*}-1}}{\Gamma\left(p^{*}\right)}|\kappa(m, z(m))| \mathrm{d} m \\
& +\frac{\tau \Gamma\left(q^{*}+2\right)}{\eta^{q^{*}+1}} \int_{0}^{\eta} \frac{(\eta-m)^{p^{*}+q^{*}-1}}{\Gamma\left(p^{*}+q^{*}\right)}|\kappa(m, z(m))| \mathrm{d} m \\
& +\frac{\tau \Gamma\left(q^{*}+2\right)\left|\eta-\eta^{q^{*}+2}\right|}{\left(\eta^{q^{*}+2}-\eta^{q^{*}+1}\right)} \int_{0}^{1} \frac{(1-m)^{p^{*}+q^{*}-1}}{\Gamma\left(p^{*}+q^{*}\right)}|\kappa(m, z(m))| \mathrm{d} m \\
& +\frac{\tau^{2} \Gamma\left(q^{*}+3\right)}{(\eta-1)} \int_{0}^{1} \frac{(1-m)^{p^{*}+q^{*}-1}}{\Gamma\left(p^{*}+q^{*}\right)}|\kappa(m, z(m))| \mathrm{d} m \\
& +\frac{\tau^{2} \Gamma\left(q^{*}+3\right)}{\left(\eta^{q^{*}+2}-\eta^{q^{*}+1}\right)} \int_{0}^{\eta} \frac{(\eta-m)^{p^{*}+q^{*}-1}}{\Gamma\left(p^{*}+q^{*}\right)}|\kappa(m, z(m))| \mathrm{d} m \\
\leq & \frac{\tau^{p^{*}}}{\Gamma\left(p^{*}+1\right)} \sigma(m) \zeta(\|z\|)+\frac{\tau \Gamma\left(q^{*}+2\right) \eta^{p^{*}-1}}{\Gamma\left(p^{*}+q^{*}+1\right)} \sigma(m) \zeta(\|z\|) \\
& +\frac{\tau \Gamma\left(q^{*}+2\right)\left|\eta-\eta^{q^{*}+2}\right|}{\left(\eta^{q^{*}+2}-\eta^{q^{*}+1}\right) \Gamma\left(p^{*}+q^{*}+1\right)} \sigma(m) \zeta(\|z\|) \\
& +\frac{\tau^{2} \Gamma\left(q^{*}+3\right)}{(\eta-1) \Gamma\left(p^{*}+q^{*}+1\right)} \sigma(m) \zeta(\|z\|) \\
& +\frac{\tau^{2} \Gamma\left(q^{*}+3\right) \eta^{p^{*}+q^{*}}}{\left(\eta^{q^{*}+2}-\eta^{q^{*}+1}\right) \Gamma\left(p^{*}+q^{*}+1\right)} \sigma(m) \zeta(\|z\|)
\end{aligned}
$$

for each $z \in \bar{V}_{\epsilon}(0)$. Hence $\left\|A_{2} z\right\| \leq M^{*} \zeta(\|z\|) \Delta$, where $\Delta$ is given in (9). This implies that the set $A_{2}\left(\bar{V}_{\epsilon}(0)\right)$ is uniformly bounded in the space $\mathcal{X}$.

In the following, the equicontinuity of the operator $A_{2}$ is investigated. For this aim, assume that $\tau_{1}, \tau_{2} \in[0,1]$ with $\tau_{1}<\tau_{2}$. Then we have

$$
\begin{aligned}
\left|\left(A_{2} z\right)\left(\tau_{2}\right)-\left(A_{2} z\right)\left(\tau_{1}\right)\right|= & \mid \int_{0}^{\tau_{2}} \frac{\left(\tau_{2}-m\right)^{p^{*}-1}}{\Gamma\left(p^{*}\right)} \kappa(m, z(m)) \mathrm{d} m \\
& -\int_{0}^{\tau_{1}} \frac{\left(\tau_{1}-m\right)^{p^{*}-1}}{\Gamma\left(p^{*}\right)} \kappa(m, z(m)) \mathrm{d} m \\
& -\frac{\left(\tau_{2}-\tau_{1}\right) \Gamma\left(q^{*}+2\right)}{\eta^{q^{*}+1}} \int_{0}^{\eta} \frac{(\eta-m)^{p^{*}+q^{*}-1}}{\Gamma\left(p^{*}+q^{*}\right)} \kappa(m, z(m)) \mathrm{d} m
\end{aligned}
$$




$$
\begin{aligned}
& +\frac{\left(\tau_{2}-\tau_{1}\right) \Gamma\left(q^{*}+2\right)\left|\eta-\eta^{q^{*}+2}\right|}{\left(\eta^{q^{*}+2}-\eta^{q^{*}+1}\right)} \\
& \times \int_{0}^{1} \frac{(1-m)^{p^{*}+q^{*}-1}}{\Gamma\left(p^{*}+q^{*}\right)} \kappa(m, z(m)) \mathrm{d} m \\
& +\frac{\left(\tau_{2}^{2}-\tau_{1}^{2}\right) \Gamma\left(q^{*}+3\right)}{(\eta-1)} \int_{0}^{1} \frac{(1-m)^{p^{*}+q^{*}-1}}{\Gamma\left(p^{*}+q^{*}\right)} \kappa(m, z(m)) \mathrm{d} m \\
& -\frac{\left(\tau_{2}^{2}-\tau_{1}^{2}\right) \Gamma\left(q^{*}+3\right)}{\left(\eta^{q^{*}+2}-\eta^{q^{*}+1}\right)} \int_{0}^{\eta} \frac{(\eta-m)^{p^{*}+q^{*}-1}}{\Gamma\left(p^{*}+q^{*}\right)} \kappa(m, z(m)) \mathrm{d} m \mid \\
& \leq M^{*} \zeta(\|z\|)\left[\int_{0}^{\tau_{1}}\left(\frac{\left(\tau_{2}-m\right)^{p^{*}-1}}{\Gamma\left(p^{*}\right)}-\frac{\left(\tau_{1}-m\right)^{p^{*}-1}}{\Gamma\left(p^{*}\right)}\right) \mathrm{d} m\right. \\
& +\int_{\tau_{1}}^{\tau_{2}} \frac{\left(\tau_{2}-m\right)^{p^{*}-1}}{\Gamma\left(p^{*}\right)} \mathrm{d} m \\
& +\frac{\left(\tau_{2}-\tau_{1}\right) \Gamma\left(q^{*}+2\right)}{\eta^{q^{*}+1}} \int_{0}^{\eta} \frac{(\eta-m)^{p^{*}+q^{*}-1}}{\Gamma\left(p^{*}+q^{*}\right)} \mathrm{d} m \\
& +\frac{\left(\tau_{2}-\tau_{1}\right) \Gamma\left(q^{*}+2\right)\left|\eta-\eta^{q^{*}+2}\right|}{\left(\eta^{q^{*}+2}-\eta^{q^{*}+1}\right)} \int_{0}^{1} \frac{(1-m)^{p^{*}+q^{*}-1}}{\Gamma\left(p^{*}+q^{*}\right)} \mathrm{d} m \\
& +\frac{\left(\tau_{2}^{2}-\tau_{1}^{2}\right) \Gamma\left(q^{*}+3\right)}{(\eta-1)} \int_{0}^{1} \frac{(1-m)^{p^{*}+q^{*}-1}}{\Gamma\left(p^{*}+q^{*}\right)} \mathrm{d} m \\
& \left.+\frac{\left(\tau_{2}^{2}-\tau_{1}^{2}\right) \Gamma\left(q^{*}+3\right)}{\left(\eta^{q^{*}+2}-\eta^{q^{*}+1}\right)} \int_{0}^{\eta} \frac{(\eta-m)^{p^{*}+q^{*}-1}}{\Gamma\left(p^{*}+q^{*}\right)} \mathrm{d} m\right] .
\end{aligned}
$$

We observe that the right-hand side of the inequalities converges to zero independently of $z \in \bar{V}_{\epsilon}(0)$ as $\tau_{1} \rightarrow \tau_{2}$. Hence $A_{2}$ is equicontinuous. Thus, by using the Arzela-Ascoli theorem, we conclude that the operator $A_{2}$ has the complete continuity property on $\bar{V}_{\epsilon}(0)$.

On the other hand, since by condition (H3) we have

$$
\begin{aligned}
M= & \left\|A_{2}\left(\bar{V}_{\epsilon}(0)\right)\right\|_{\mathcal{X}}=\sup \left\{\left|A_{2} z\right|: z \in \bar{V}_{\epsilon}(0)\right\} \\
= & M^{*} \zeta(\|z\|)\left[\frac{1}{\Gamma\left(p^{*}+1\right)}+\frac{\Gamma\left(q^{*}+2\right) \eta^{p^{*}-1}}{\Gamma\left(p^{*}+q^{*}+1\right)}+\frac{\Gamma\left(q^{*}+2\right)\left|\eta-\eta^{q^{*}+2}\right|}{\left(\eta^{q^{*}+2}-\eta^{q^{*}+1}\right) \Gamma\left(p^{*}+q^{*}+1\right)}\right. \\
& \left.+\frac{\Gamma\left(q^{*}+3\right)}{(\eta-1) \Gamma\left(p^{*}+q^{*}+1\right)}+\frac{\Gamma\left(q^{*}+3\right) \eta^{p^{*}+q^{*}}}{\left(\eta^{q^{*}+2}-\eta^{q^{*}+1}\right) \Gamma\left(p^{*}+q^{*}+1\right)}\right] \\
= & M^{*} \zeta(\|z\|) \Delta .
\end{aligned}
$$

So, letting $k^{*}=L^{*}$, we get $M^{*} k^{*}<1$, and consequently, one can observe that all assumptions of Theorem 2.1 hold for both $A_{1}$ and $A_{2}$. Hence, one of conditions, condition (i) or condition (ii), of Theorem 2.1 holds. For some $\lambda \in(0,1)$, let us assume that $z$ satisfies the operator equation $z=\lambda A_{1} z A_{2} z$ so that $\|z\|=\epsilon$. Now, one can write

$$
\begin{aligned}
|z(\tau)|= & \lambda\left|\left(A_{1} z\right)(\tau)\right|\left|\left(A_{2} z\right)(\tau)\right| \\
= & \lambda|\rho(\tau, z(\tau))| \mid \int_{0}^{\tau} \frac{(\tau-m)^{p^{*}-1}}{\Gamma\left(p^{*}\right)} \kappa(m, z(m)) \mathrm{d} m \\
& -\frac{\tau \Gamma\left(q^{*}+2\right)}{\eta^{q^{*}+1}} \int_{0}^{\eta} \frac{(\eta-m)^{p^{*}+q^{*}-1}}{\Gamma\left(p^{*}+q^{*}\right)} \kappa(m, z(m)) \mathrm{d} m
\end{aligned}
$$




$$
\begin{aligned}
+ & \frac{\tau \Gamma\left(q^{*}+2\right)\left|\eta-\eta^{q^{*}+2}\right|}{\left(\eta^{q^{*}+2}-\eta^{q^{*}+1}\right)} \int_{0}^{1} \frac{(1-m)^{p^{*}+q^{*}-1}}{\Gamma\left(p^{*}+q^{*}\right)} \kappa(m, z(m)) \mathrm{d} m \\
+ & \frac{\tau^{2} \Gamma\left(q^{*}+3\right)}{((\eta-1)} \int_{0}^{1} \frac{(1-m)^{p^{*}+q^{*}-1}}{\Gamma\left(p^{*}+q^{*}\right)} \kappa(m, z(m)) \mathrm{d} m \\
& -\frac{\tau^{2} \Gamma\left(q^{*}+3\right)}{\left(\eta^{q^{*}+2}-\eta^{q^{*}+1}\right)} \int_{0}^{\eta} \frac{(\eta-m)^{p^{*}+q^{*}-1}}{\Gamma\left(p^{*}+q^{*}\right)} \kappa(m, z(m)) \mathrm{d} m \mid \\
\leq & (|\rho(\tau, z(\tau))-\rho(\tau, 0)|+|\rho(\tau, 0)|)\left(\int_{0}^{\tau} \frac{(\tau-m)^{p^{*}-1}}{\Gamma\left(p^{*}\right)}|\kappa(m, z(m))| \mathrm{d} m\right. \\
& +\frac{\tau \Gamma\left(q^{*}+2\right)}{\eta^{q^{*}+1}} \int_{0}^{\eta} \frac{(\eta-m)^{p^{*}+q^{*}-1}}{\Gamma\left(p^{*}+q^{*}\right)}|\kappa(m, z(m))| \mathrm{d} m \\
& +\frac{\tau \Gamma\left(q^{*}+2\right)\left|\eta-\eta^{q^{*}+2}\right|}{\left(\eta^{q^{*}+2}-\eta^{q^{*}+1}\right)} \int_{0}^{1} \frac{(1-m)^{p^{*}+q^{*}-1}}{\Gamma\left(p^{*}+q^{*}\right)}|\kappa(m, z(m))| \mathrm{d} m \\
& +\frac{\tau^{2} \Gamma\left(q^{*}+3\right)}{(\eta-1)} \int_{0}^{1} \frac{(1-m)^{p^{*}+q^{*}-1}}{\Gamma\left(p^{*}+q^{*}\right)}|\kappa(m, z(m))| \mathrm{d} m \\
& \left.+\frac{\tau^{2} \Gamma\left(q^{*}+3\right)}{\left(\eta^{q^{*}+2}-\eta^{q^{*}+1}\right)} \int_{0}^{\eta} \frac{(\eta-m)^{p^{*}+q^{*}-1}}{\Gamma\left(p^{*}+q^{*}\right)}|\kappa(m, z(m))| \mathrm{d} m\right) \\
\leq & \left(L(x)|z(\tau)|+F^{*}\right) \Delta M^{*} \zeta(\|z\|) \\
\leq & \left(L^{*}\|z\|+F^{*}\right) \Delta M^{*} \zeta(\|z\|) .
\end{aligned}
$$

In this case, we see that

$$
\epsilon \leq \frac{F^{*} \Delta M^{*} \zeta(\|z\|)}{1-L^{*} \Delta M^{*} \zeta(\|z\|)},
$$

which is a contradiction to (8). This shows that condition (ii) of Theorem 2.1 is impossible. Therefore, condition (i) of Theorem 2.1 holds and the fractional hybrid BVP (1)-(2) has a solution on $\bar{V}_{\epsilon}(0)$.

Now, we are ready to prove the existence result for the hybrid differential inclusion of the fractional order (3) with three-point integral hybrid conditions (2).

Definition 3.3 The absolutely continuous function $z:[0,1] \rightarrow \mathbb{R}$ is called a solution function for the fractional hybrid BVP (3)-(2) if there is an integrable function $v \in \mathcal{L}^{1}([0,1], \mathbb{R})$ with $v(\tau) \in \mathcal{H}(\tau, z(\tau))$ for almost all $\tau \in[0,1]$ such that $z(0)=0$,

$$
\begin{aligned}
& \left.\left(\frac{z(\tau)}{\rho(\tau, z(\tau))}\right)\right|_{\tau=0}+\left.\mathcal{J}_{0}^{q^{*}}\left(\frac{z(\tau)}{\rho(\tau, z(\tau))}\right)\right|_{\tau=\eta}=0, \\
& \left.\left(\frac{z(\tau)}{\rho(\tau, z(\tau))}\right)\right|_{\tau=0}+\left.\mathcal{J}_{0}^{q^{*}}\left(\frac{z(\tau)}{\rho(\tau, z(\tau))}\right)\right|_{\tau=1}=0
\end{aligned}
$$

and for all $\tau \in[0,1]$,

$$
\begin{aligned}
z(\tau)= & \rho(\tau, z(\tau))\left[\int_{0}^{\tau} \frac{(\tau-m)^{p^{*}-1}}{\Gamma\left(p^{*}\right)} v(m) \mathrm{d} m-\frac{\tau \Gamma\left(q^{*}+2\right)}{\eta^{q^{*}+1}} \int_{0}^{\eta} \frac{(\eta-m)^{p^{*}+q^{*}-1}}{\Gamma\left(p^{*}+q^{*}\right)} v(m) \mathrm{d} m\right. \\
& +\frac{\tau \Gamma\left(q^{*}+2\right)\left(\eta-\eta^{q^{*}+2}\right)}{\left(\eta^{q^{*}+2}-\eta^{q^{*}+1}\right)} \int_{0}^{1} \frac{(1-m)^{p^{*}+q^{*}-1}}{\Gamma\left(p^{*}+q^{*}\right)} v(m) \mathrm{d} m
\end{aligned}
$$




$$
\begin{aligned}
& +\frac{\tau^{2} \Gamma\left(q^{*}+3\right)}{(\eta-1)} \int_{0}^{1} \frac{(1-m)^{p^{*}+q^{*}-1}}{\Gamma\left(p^{*}+q^{*}\right)} v(m) \mathrm{d} m \\
& \left.-\frac{\tau^{2} \Gamma\left(q^{*}+3\right)}{\left(\eta^{q^{*}+2}-\eta^{q^{*}+1}\right)} \int_{0}^{\eta} \frac{(\eta-m)^{p^{*}+q^{*}-1}}{\Gamma\left(p^{*}+q^{*}\right)} v(m) \mathrm{d} m\right] .
\end{aligned}
$$

\section{Theorem 3.4 Assume that:}

(N1) There is a bounded mapping $L:[0,1] \rightarrow \mathbb{R}^{+}$provided that, for every member $z_{1}, z_{2} \in \mathbb{R}$ and $\tau \in[0,1]$

$$
\left|\rho\left(\tau, z_{1}(\tau)\right)-\rho\left(\tau, z_{2}(\tau)\right)\right| \leq L(\tau)\left|z_{1}(\tau)-z_{2}(\tau)\right| .
$$

(N2) The multifunction $\mathcal{H}:[0,1] \times \mathbb{R} \rightarrow \mathcal{P}_{c p, c v}(\mathbb{R})$ has the $\mathcal{L}^{1}$-Caratheodory property.

(N3) There is a positive mapping $s \in \mathcal{L}^{1}\left([0,1], \mathbb{R}^{+}\right)$such that, for all $z \in \mathbb{R}$,

$$
\|\mathcal{H}(\tau, z)\|=\sup \{|v|: v \in \mathcal{H}(\tau, z(\tau))\} \leq s(\tau)
$$

for almost all $\tau \in[0,1]$ and $\|s\|_{\mathcal{L}^{1}}=\int_{0}^{1}|s(\tau)| \mathrm{d} \tau$.

(N4) There is a positive number $\tilde{\epsilon} \in \mathbb{R}$ such that

$$
\tilde{\epsilon}>\frac{F^{*} \Delta\|s\|_{\mathcal{L}^{1}}}{1-L^{*} \Delta\|s\|_{\mathcal{L}^{1}}}
$$

where $F^{*}=\sup _{\tau \in[0,1]}|\rho(\tau, 0)|$ and $L^{*}=\sup _{\tau \in[0,1]}|L(\tau)|$.

If $L^{*} \Delta\|s\|_{\mathcal{L}^{1}}<\frac{1}{2}$, then the fractional hybrid differential inclusion (3) with three-point integral hybrid conditions (2) has at least one solution function on $[0,1]$.

Proof First, we define an operator $\mathcal{N}: \mathcal{X} \rightarrow \mathcal{P}(\mathcal{X})$ as follows:

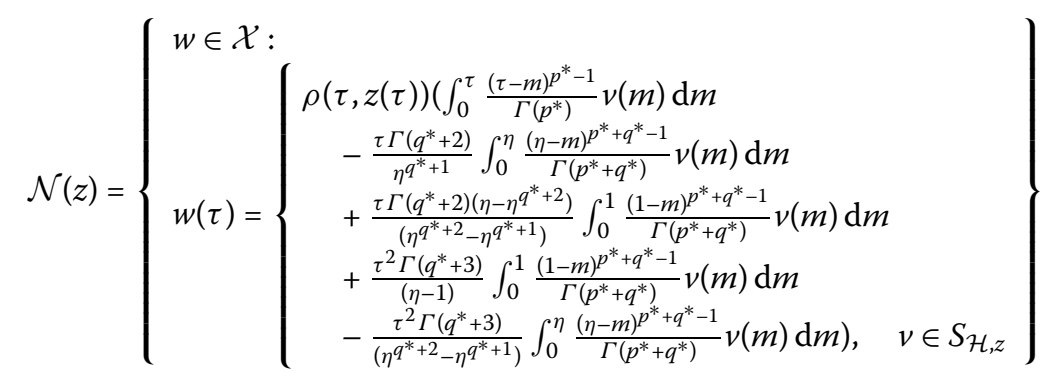

for all $\tau \in[0,1]$. This implies that the fractional hybrid boundary value problem (3)-(2) transforms into a fixed point theorem. Now, we define a single-valued mapping $A_{1}: \mathcal{X} \rightarrow$ $\mathcal{X}$ by

$$
\left(A_{1} z\right)(\tau)=\rho(\tau, z(\tau)), \quad \tau \in[0,1]
$$


and a multi-valued mapping $A_{2}: \mathcal{X} \rightarrow \mathcal{P}(\mathcal{X})$ by

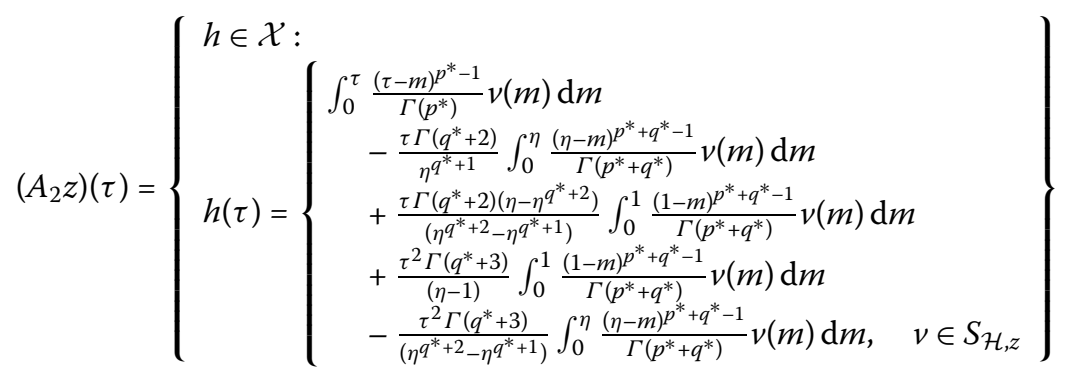

for all $\tau \in[0,1]$. It is obvious that $\mathcal{N}(z)=A_{1} z A_{2} z$. We will prove that both $A_{1}$ and $A_{2}$ satisfy all the assumptions of Theorem 2.3. As a first step, by using assumption (N1), we show that $A_{1}$ is Lipschitz on $\mathcal{X}$, but because of the similarity of the proof for the operator $A_{1}$ in Theorem 3.2, we omit the proof.

In the next step, we prove that $A_{2}$ is convex-valued. For this aim, let $z_{1}, z_{2} \in A_{2} z$. Then, we choose $v_{1}, v_{2} \in S_{\mathcal{H}, z}$ such that

$$
\begin{aligned}
z_{i}(\tau)= & \int_{0}^{\tau} \frac{(\tau-m)^{p^{*}-1}}{\Gamma\left(p^{*}\right)} v_{i}(m) \mathrm{d} m-\frac{\tau \Gamma\left(q^{*}+2\right)}{\eta^{q^{*}+1}} \int_{0}^{\eta} \frac{(\eta-m)^{p^{*}+q^{*}-1}}{\Gamma\left(p^{*}+q^{*}\right)} v_{i}(m) \mathrm{d} m \\
& +\frac{\tau \Gamma\left(q^{*}+2\right)\left(\eta-\eta^{q^{*}+2}\right)}{\left(\eta^{q^{*}+2}-\eta^{q^{*}+1}\right)} \int_{0}^{1} \frac{(1-m)^{p^{*}+q^{*}-1}}{\Gamma\left(p^{*}+q^{*}\right)} v_{i}(m) \mathrm{d} m \\
& +\frac{\tau^{2} \Gamma\left(q^{*}+3\right)}{(\eta-1)} \int_{0}^{1} \frac{(1-m)^{p^{*}+q^{*}-1}}{\Gamma\left(p^{*}+q^{*}\right)} v_{i}(m) \mathrm{d} m \\
& -\frac{\tau^{2} \Gamma\left(q^{*}+3\right)}{\left(\eta^{q^{*}+2}-\eta^{q^{*}+1}\right)} \int_{0}^{\eta} \frac{(\eta-m)^{p^{*}+q^{*}-1}}{\Gamma\left(p^{*}+q^{*}\right)} v_{i}(m) \mathrm{d} m \quad(i=1,2)
\end{aligned}
$$

for almost all $\tau \in[0,1]$. For any $\mu \in(0,1)$, we obtain

$$
\begin{aligned}
\mu z_{1}(\tau) & +(1-\mu) z_{2}(\tau) \\
= & \int_{0}^{\tau} \frac{(\tau-m)^{p^{*}-1}}{\Gamma\left(p^{*}\right)}\left[\mu v_{1}(m)+(1-\mu) v_{2}(m)\right] \mathrm{d} m \\
& -\frac{\tau \Gamma\left(q^{*}+2\right)}{\eta^{q^{*}+1}} \int_{0}^{\eta} \frac{(\eta-m)^{p^{*}+q^{*}-1}}{\Gamma\left(p^{*}+q^{*}\right)}\left[\mu v_{1}(m)+(1-\mu) v_{2}(m)\right] \mathrm{d} m \\
& +\frac{\tau \Gamma\left(q^{*}+2\right)\left(\eta-\eta^{q^{*}+2}\right)}{\left(\eta^{q^{*}+2}-\eta^{q^{*}+1}\right)} \int_{0}^{1} \frac{(1-m)^{p^{*}+q^{*}-1}}{\Gamma\left(p^{*}+q^{*}\right)}\left[\mu v_{1}(m)+(1-\mu) v_{2}(m)\right] \mathrm{d} m \\
& +\frac{\tau^{2} \Gamma\left(q^{*}+3\right)}{(\eta-1)} \int_{0}^{1} \frac{(1-m)^{p^{*}+q^{*}-1}}{\Gamma\left(p^{*}+q^{*}\right)}\left[\mu v_{1}(m)+(1-\mu) v_{2}(m)\right] \mathrm{d} m \\
& -\frac{\tau^{2} \Gamma\left(q^{*}+3\right)}{\left(\eta^{q^{*}+2}-\eta^{q^{*}+1}\right)} \int_{0}^{\eta} \frac{(\eta-m)^{p^{*}+q^{*}-1}}{\Gamma\left(p^{*}+q^{*}\right)}\left[\mu v_{1}(m)+(1-\mu) v_{2}(m)\right] \mathrm{d} m
\end{aligned}
$$

for almost all $\tau \in[0,1]$. Since the multifunction $\mathcal{H}$ has convex values, thus $S_{\mathcal{H}, z}$ is convexvalued and therefore, for each $\tau \in[0,1]$, we get $\mu v_{1}(\tau)+(1-\mu) v_{2}(\tau) \in S_{\mathcal{H}, z}$. Consequently, $A_{2} z$ is a convex set for each $z \in \mathcal{X}$.

To prove the complete continuity of the operator $A_{2}$, we must prove that $A_{2}(\mathcal{X})$ is an equicontinuous and uniformly bounded set. To do this, we prove that $A_{2}$ maps all 
bounded sets into bounded subsets of the space $\mathcal{X}$. For a positive number $\epsilon^{*} \in \mathbb{R}$, consider a bounded ball $V_{\epsilon^{*}}=\left\{z \in \mathcal{X}:\|z\|_{\mathcal{X}} \leq \epsilon^{*}\right\}$ in $\mathcal{X}$. For every member $\tau \in V_{\epsilon^{*}}$ and $h \in A_{2} z$, there is a function $v \in S_{\mathcal{H}, z}$ provided that

$$
\begin{aligned}
h(\tau)= & \int_{0}^{\tau} \frac{(\tau-m)^{p^{*}-1}}{\Gamma\left(p^{*}\right)} v(m) \mathrm{d} m-\frac{\tau \Gamma\left(q^{*}+2\right)}{\eta^{q^{*}+1}} \int_{0}^{\eta} \frac{(\eta-m)^{p^{*}+q^{*}-1}}{\Gamma\left(p^{*}+q^{*}\right)} v(m) \mathrm{d} m \\
& +\frac{\tau \Gamma\left(q^{*}+2\right)\left(\eta-\eta^{q^{*}+2}\right)}{\left(\eta^{q^{*}+2}-\eta^{q^{*}+1}\right)} \int_{0}^{1} \frac{(1-m)^{p^{*}+q^{*}-1}}{\Gamma\left(p^{*}+q^{*}\right)} v(m) \mathrm{d} m \\
& +\frac{\tau^{2} \Gamma\left(q^{*}+3\right)}{(\eta-1)} \int_{0}^{1} \frac{(1-m)^{p^{*}+q^{*}-1}}{\Gamma\left(p^{*}+q^{*}\right)} v(m) \mathrm{d} m \\
& -\frac{\tau^{2} \Gamma\left(q^{*}+3\right)}{\left(\eta^{q^{*}+2}-\eta^{q^{*}+1}\right)} \int_{0}^{\eta} \frac{(\eta-m)^{p^{*}+q^{*}-1}}{\Gamma\left(p^{*}+q^{*}\right)} v(m) \mathrm{d} m
\end{aligned}
$$

for all $\tau \in[0,1]$. Then we have

$$
\begin{aligned}
|h(\tau)| \leq & \int_{0}^{\tau} \frac{(\tau-m)^{p^{*}-1}}{\Gamma\left(p^{*}\right)}|v(m)| \mathrm{d} m+\frac{\tau \Gamma\left(q^{*}+2\right)}{\eta^{q^{*}+1}} \int_{0}^{\eta} \frac{(\eta-m)^{p^{*}+q^{*}-1}}{\Gamma\left(p^{*}+q^{*}\right)}|v(m)| \mathrm{d} m \\
& +\frac{\tau \Gamma\left(q^{*}+2\right)\left|\eta-\eta^{q^{*}+2}\right|}{\left(\eta^{q^{*}+2}-\eta^{q^{*}+1}\right)} \int_{0}^{1} \frac{(1-m)^{p^{*}+q^{*}-1}}{\Gamma\left(p^{*}+q^{*}\right)}|v(m)| \mathrm{d} m \\
& +\frac{\tau^{2} \Gamma\left(q^{*}+3\right)}{(\eta-1)} \int_{0}^{1} \frac{(1-m)^{p^{*}+q^{*}-1}}{\Gamma\left(p^{*}+q^{*}\right)}|v(m)| \mathrm{d} m \\
& +\frac{\tau^{2} \Gamma\left(q^{*}+3\right)}{\left(\eta^{q^{*}+2}-\eta^{q^{*}+1}\right)} \int_{0}^{\eta} \frac{(\eta-m)^{p^{*}+q^{*}-1}}{\Gamma\left(p^{*}+q^{*}\right)}|v(m)| \mathrm{d} m \\
\leq & \int_{0}^{\tau} \frac{(\tau-m)^{p^{*}-1}}{\Gamma\left(p^{*}\right)} s(m) \mathrm{d} m+\frac{\tau \Gamma\left(q^{*}+2\right)}{\eta^{q^{*}+1}} \int_{0}^{\eta} \frac{(\eta-m)^{p^{*}+q^{*}-1}}{\Gamma\left(p^{*}+q^{*}\right)}|v(m)| \mathrm{d} m \\
& +\frac{\tau \Gamma\left(q^{*}+2\right)\left|\eta-\eta^{q^{*}+2}\right|}{\left(\eta^{q^{*}+2}-\eta^{q^{*}+1}\right)} \int_{0}^{1} \frac{(1-m)^{p^{*}+q^{*}-1}}{\Gamma\left(p^{*}+q^{*}\right)} s(m) \mathrm{d} m \\
& +\frac{\tau^{2} \Gamma\left(q^{*}+3\right)}{(\eta-1)} \int_{0}^{1} \frac{(1-m)^{p^{*}+q^{*}-1}}{\Gamma\left(p^{*}+q^{*}\right)} s(m) \mathrm{d} m \\
& +\frac{\tau^{2} \Gamma\left(q^{*}+3\right)}{\left(\eta^{q^{*}+2}-\eta^{q^{*}+1}\right)} \int_{0}^{\eta} \frac{(\eta-m)^{p^{*}+q^{*}-1}}{\Gamma\left(p^{*}+q^{*}\right)} s(m) \mathrm{d} m \\
\leq & {\left[\frac{1}{\Gamma\left(p^{*}+1\right)}+\frac{\Gamma\left(q^{*}+2\right) \eta^{p^{*}-1}}{\Gamma\left(p^{*}+q^{*}+1\right)}+\frac{\Gamma\left(q^{*}+2\right)\left|\eta-\eta^{q^{*}+2}\right|}{\left(\eta^{q^{*}+2}-\eta^{q^{*}+1}\right) \Gamma\left(p^{*}+q^{*}+1\right)}\right.} \\
& \left.+\frac{\Gamma\left(q^{*}+3\right)}{(\eta-1) \Gamma\left(p^{*}+q^{*}+1\right)}+\frac{\Gamma\left(q^{*}+3\right) \eta^{p^{*}+q^{*}}}{\left(\eta^{q^{*}+2}-\eta^{q^{*}+1}\right) \Gamma\left(p^{*}+q^{*}+1\right)}\right]\|s\|_{\mathcal{L}^{1}} \\
= & \Delta\|s\| \mathcal{L}^{1},
\end{aligned}
$$

where $\Delta$ is given in (9). Thus $\|h\| \leq \Delta\|s\|_{\mathcal{L}^{1}}$ and we observe that the set $A_{2}(\mathcal{X})$ is uniformly bounded.

Now, we continue the proof in the next step. We show that if the domain of the operator $A_{2}$ is bounded sets, then the range of this operator is equicontinuous subsets of $\mathcal{X}$. Assume 
that $z \in V_{\epsilon^{*}}$. For $h \in A_{2} z$, we choose $v \in S_{\mathcal{H}, z}$ such that

$$
\begin{aligned}
h(\tau)= & \int_{0}^{\tau} \frac{(\tau-m)^{p^{*}-1}}{\Gamma\left(p^{*}\right)} v(m) \mathrm{d} m-\frac{\tau \Gamma\left(q^{*}+2\right)}{\eta^{q^{*}+1}} \int_{0}^{\eta} \frac{(\eta-m)^{p^{*}+q^{*}-1}}{\Gamma\left(p^{*}+q^{*}\right)} v(m) \mathrm{d} m \\
& +\frac{\tau \Gamma\left(q^{*}+2\right)\left(\eta-\eta^{q^{*}+2}\right)}{\left(\eta^{q^{*}+2}-\eta^{q^{*}+1}\right)} \int_{0}^{1} \frac{(1-m)^{p^{*}+q^{*}-1}}{\Gamma\left(p^{*}+q^{*}\right)} v(m) \mathrm{d} m \\
& +\frac{\tau^{2} \Gamma\left(q^{*}+3\right)}{(\eta-1)} \int_{0}^{1} \frac{(1-m)^{p^{*}+q^{*}-1}}{\Gamma\left(p^{*}+q^{*}\right)} v(m) \mathrm{d} m \\
& -\frac{\tau^{2} \Gamma\left(q^{*}+3\right)}{\left(\eta^{q^{*}+2}-\eta^{q^{*}+1}\right)} \int_{0}^{\eta} \frac{(\eta-m)^{p^{*}+q^{*}-1}}{\Gamma\left(p^{*}+q^{*}\right)} v(m) \mathrm{d} m
\end{aligned}
$$

for all $\tau \in[0,1]$. Then, for arbitrary variables $\tau_{1}, \tau_{2} \in[0,1]$ with $\tau_{1}<\tau_{2}$, we have

$$
\begin{aligned}
\left|h\left(\tau_{2}\right)-h\left(\tau_{1}\right)\right| \leq & \left|\int_{0}^{\tau_{2}} \frac{\left(\tau_{2}-m\right)^{p^{*}-1}}{\Gamma\left(p^{*}\right)} v(m) \mathrm{d} m-\int_{0}^{\tau_{1}} \frac{\left(\tau_{1}-m\right)^{p^{*}-1}}{\Gamma\left(p^{*}\right)} v(m) \mathrm{d} m\right| \\
& +\frac{\left(\tau_{2}-\tau_{1}\right) \Gamma\left(q^{*}+2\right)}{\eta^{q^{*}+1}} \int_{0}^{\eta} \frac{(\eta-m)^{p^{*}+q^{*}-1}}{\Gamma\left(p^{*}+q^{*}\right)}|v(m)| \mathrm{d} m \\
& +\frac{\left(\tau_{2}-\tau_{1}\right) \Gamma\left(q^{*}+2\right)\left|\eta-\eta^{q^{*}+2}\right|}{\left(\eta^{q^{*}+2}-\eta^{q^{*}+1}\right)} \int_{0}^{1} \frac{(1-m)^{p^{*}+q^{*}-1}}{\Gamma\left(p^{*}+q^{*}\right)}|v(m)| \mathrm{d} m \\
& +\frac{\left(\tau_{2}^{2}-\tau_{1}^{2}\right) \Gamma\left(q^{*}+3\right)}{(\eta-1)} \int_{0}^{1} \frac{(1-m)^{p^{*}+q^{*}-1}}{\Gamma\left(p^{*}+q^{*}\right)}|v(m)| \mathrm{d} m \\
& +\frac{\left(\tau_{2}^{2}-\tau_{1}^{2}\right) \Gamma\left(q^{*}+3\right)}{\left(\eta^{q^{*}+2}-\eta^{q^{*}+1}\right)} \int_{0}^{\eta} \frac{(\eta-m)^{p^{*}+q^{*}-1}}{\Gamma\left(p^{*}+q^{*}\right)}|v(m)| \mathrm{d} m \\
\leq & \int_{0}^{\tau_{1}}\left(\frac{\left[\left(\tau_{2}-m\right)^{p^{*}-1}-\left(\tau_{1}-m\right)^{p^{*}-1}\right]}{\Gamma\left(p^{*}\right)}\right) \mathrm{d}(m) \\
& +\int_{\tau_{1}}^{\tau_{2}} \frac{\left(\tau_{2}-m\right)^{p^{*}-1}}{\Gamma\left(p^{*}\right)} s(m) \mathrm{d} m \\
& +\frac{\left(\tau_{2}-\tau_{1}\right) \Gamma\left(q^{*}+2\right)}{\eta^{q^{*}+1}} \int_{0}^{\eta} \frac{(\eta-m)^{p^{*}+q^{*}-1}}{\Gamma\left(p^{*}+q^{*}\right)} s(m) \mathrm{d} m \\
& +\frac{\left(\tau_{2}-\tau_{1}\right) \Gamma\left(q^{*}+2\right)\left|\eta-\eta^{q^{*}+2}\right|}{\left(\eta^{q^{*}+2}-\eta^{q^{*}+1}\right)} \int_{0}^{1} \frac{(1-m)^{p^{*}+q^{*}-1}}{\Gamma\left(p^{*}+q^{*}\right)} s(m) \mathrm{d} m \\
& +\frac{\left(\tau_{2}^{2}-\tau_{1}^{2}\right) \Gamma\left(q^{*}+3\right)}{(\eta-1)} \int_{0}^{1} \frac{(1-m)^{p^{*}+q^{*}-1}}{\Gamma\left(p^{*}+q^{*}\right)} s(m) \mathrm{d} m \\
& +\frac{\left(\tau_{2}^{2}-\tau_{1}^{2}\right) \Gamma\left(q^{*}+3\right)}{\left(\eta^{q^{*}+2}-\eta^{q^{*}+1}\right)} \int_{0}^{\eta} \frac{(\eta-m)^{p^{*}+q^{*}-1}}{\Gamma\left(p^{*}+q^{*}\right)} s(m) \mathrm{d} m .
\end{aligned}
$$

It is seen that the right-hand side of the above inequalities converges to zero independently of $z \in V_{\epsilon^{*}}$ as $\tau_{2} \rightarrow \tau_{1}$. Hence by using the Arzela-Ascoli theorem, it is deduced that the operator $A_{2}: C([0,1], \mathbb{R}) \rightarrow \mathcal{P}(C([0,1], \mathbb{R}))$ has the complete continuity property.

Now, we show that $A_{2}$ has a closed graph since this implies that the completely continuous operator $A_{2}$ is upper semi-continuous. For this aim, let $z_{n} \in V_{\epsilon^{*}}$ and $h_{n} \in A_{2} z_{n}$ for all $n$ such that $z_{n} \rightarrow z^{*}$ and $h_{n} \rightarrow h^{*}$. We claim that $h^{*} \in A_{2} z^{*}$. To prove this, for each $n \geq 1$ 
and associated with $h_{n} \in A_{2} z_{n}$, we choose $v_{n} \in S_{\mathcal{H}, z_{n}}$ such that

$$
\begin{aligned}
h_{n}(\tau)= & \int_{0}^{\tau} \frac{(\tau-m)^{p^{*}-1}}{\Gamma\left(p^{*}\right)} v_{n}(m) \mathrm{d} m-\frac{\tau \Gamma\left(q^{*}+2\right)}{\eta^{q^{*}+1}} \int_{0}^{\eta} \frac{(\eta-m)^{p^{*}+q^{*}-1}}{\Gamma\left(p^{*}+q^{*}\right)} v_{n}(m) \mathrm{d} m \\
& +\frac{\tau \Gamma\left(q^{*}+2\right)\left(\eta-\eta^{q^{*}+2}\right)}{\left(\eta^{q^{*}+2}-\eta^{q^{*}+1}\right)} \int_{0}^{1} \frac{(1-m)^{p^{*}+q^{*}-1}}{\Gamma\left(p^{*}+q^{*}\right)} v_{n}(m) \mathrm{d} m \\
& +\frac{\tau^{2} \Gamma\left(q^{*}+3\right)}{(\eta-1)} \int_{0}^{1} \frac{(1-m)^{p^{*}+q^{*}-1}}{\Gamma\left(p^{*}+q^{*}\right)} v_{n}(m) \mathrm{d} m \\
& -\frac{\tau^{2} \Gamma\left(q^{*}+3\right)}{\left(\eta^{q^{*}+2}-\eta^{q^{*}+1}\right)} \int_{0}^{\eta} \frac{(\eta-m)^{p^{*}+q^{*}-1}}{\Gamma\left(p^{*}+q^{*}\right)} v_{n}(m) \mathrm{d} m
\end{aligned}
$$

for all $\tau \in[0,1]$. It is sufficient to show that there exists a function $v^{*} \in S_{\mathcal{H}, z^{*}}$ such that

$$
\begin{aligned}
h^{*}(\tau)= & \int_{0}^{\tau} \frac{(\tau-m)^{p^{*}-1}}{\Gamma\left(p^{*}\right)} v^{*}(m) \mathrm{d} m-\frac{\tau \Gamma\left(q^{*}+2\right)}{\eta^{q^{*}+1}} \int_{0}^{\eta} \frac{(\eta-m)^{p^{*}+q^{*}-1}}{\Gamma\left(p^{*}+q^{*}\right)} v^{*}(m) \mathrm{d} m \\
& +\frac{\tau \Gamma\left(q^{*}+2\right)\left(\eta-\eta^{q^{*}+2}\right)}{\left(\eta^{q^{*}+2}-\eta^{q^{*}+1}\right)} \int_{0}^{1} \frac{(1-m)^{p^{*}+q^{*}-1}}{\Gamma\left(p^{*}+q^{*}\right)} v^{*}(m) \mathrm{d} m \\
& +\frac{\tau^{2} \Gamma\left(q^{*}+3\right)}{(\eta-1)} \int_{0}^{1} \frac{(1-m)^{p^{*}+q^{*}-1}}{\Gamma\left(p^{*}+q^{*}\right)} v^{*}(m) \mathrm{d} m \\
& -\frac{\tau^{2} \Gamma\left(q^{*}+3\right)}{\left(\eta^{q^{*}+2}-\eta^{q^{*}+1}\right)} \int_{0}^{\eta} \frac{(\eta-m)^{p^{*}+q^{*}-1}}{\Gamma\left(p^{*}+q^{*}\right)} v^{*}(m) \mathrm{d} m
\end{aligned}
$$

for each $\tau \in[0,1]$. We assume that the continuous linear operator

$$
\Theta: \mathcal{L}^{1}([0,1], \mathbb{R}) \rightarrow \mathcal{X}=C([0,1], \mathbb{R})
$$

is given as follows:

$$
\begin{aligned}
\Theta(v)(\tau)=z(\tau)= & \int_{0}^{\tau} \frac{(\tau-m)^{p^{*}-1}}{\Gamma\left(p^{*}\right)} v(m) \mathrm{d} m-\frac{\tau \Gamma\left(q^{*}+2\right)}{\eta^{q^{*}+1}} \int_{0}^{\eta} \frac{(\eta-m)^{p^{*}+q^{*}-1}}{\Gamma\left(p^{*}+q^{*}\right)} v(m) \mathrm{d} m \\
& +\frac{\tau \Gamma\left(q^{*}+2\right)\left(\eta-\eta^{q^{*}+2}\right)}{\left(\eta^{q^{*}+2}-\eta^{q^{*}+1}\right)} \int_{0}^{1} \frac{(1-m)^{p^{*}+q^{*}-1}}{\Gamma\left(p^{*}+q^{*}\right)} v(m) \mathrm{d} m \\
& +\frac{\tau^{2} \Gamma\left(q^{*}+3\right)}{(\eta-1)} \int_{0}^{1} \frac{(1-m)^{p^{*}+q^{*}-1}}{\Gamma\left(p^{*}+q^{*}\right)} v(m) \mathrm{d} m \\
& -\frac{\tau^{2} \Gamma\left(q^{*}+3\right)}{\left(\eta^{q^{*}+2}-\eta^{q^{*}+1}\right)} \int_{0}^{\eta} \frac{(\eta-m)^{p^{*}+q^{*}-1}}{\Gamma\left(p^{*}+q^{*}\right)} v(m) \mathrm{d} m
\end{aligned}
$$

for each $\tau \in[0,1]$. Also, we get

$$
\begin{aligned}
\left\|h_{n}(\tau)-h^{*}(\tau)\right\|= & \| \int_{0}^{\tau} \frac{(\tau-m)^{p^{*}-1}}{\Gamma\left(p^{*}\right)}\left(v_{n}(m)-v^{*}(m)\right) \mathrm{d} m \\
& -\frac{\tau \Gamma\left(q^{*}+2\right)}{\eta^{q^{*}+1}} \int_{0}^{\eta} \frac{(\eta-m)^{p^{*}+q^{*}-1}}{\Gamma\left(p^{*}+q^{*}\right)}\left(v_{n}(m)-v^{*}(m)\right) \mathrm{d} m \\
& +\frac{\tau \Gamma\left(q^{*}+2\right)\left(\eta-\eta^{q^{*}+2}\right)}{\left(\eta^{q^{*}+2}-\eta^{q^{*}+1}\right)} \int_{0}^{1} \frac{(1-m)^{p^{*}+q^{*}-1}}{\Gamma\left(p^{*}+q^{*}\right)}\left(v_{n}(m)-v^{*}(m)\right) \mathrm{d} m
\end{aligned}
$$




$$
\begin{aligned}
& +\frac{\tau^{2} \Gamma\left(q^{*}+3\right)}{(\eta-1)} \int_{0}^{1} \frac{(1-m)^{p^{*}+q^{*}-1}}{\Gamma\left(p^{*}+q^{*}\right)}\left(v_{n}(m)-v^{*}(m)\right) \mathrm{d} m \\
& \quad-\frac{\tau^{2} \Gamma\left(q^{*}+3\right)}{\left(\eta^{q^{*}+2}-\eta^{q^{*}+1}\right)} \int_{0}^{\eta} \frac{(\eta-m)^{p^{*}+q^{*}-1}}{\Gamma\left(p^{*}+q^{*}\right)}\left(v_{n}(m)-v^{*}(m)\right) \mathrm{d} m \|
\end{aligned}
$$

as $n \rightarrow \infty$. Hence, Theorem 2.2 implies that the operator $\Theta \circ S_{\mathcal{H}}$ has a closed graph. On the other hand, since $h_{n} \in \Theta\left(S_{\mathcal{H}, z_{n}}\right)$ and $z_{n} \rightarrow z^{*}$, so there is $v^{*} \in S_{\mathcal{H}, z^{*}}$ such that

$$
\begin{aligned}
h^{*}(\tau)= & \int_{0}^{\tau} \frac{(\tau-m)^{p^{*}-1}}{\Gamma\left(p^{*}\right)} v^{*}(m) \mathrm{d} m-\frac{\tau \Gamma\left(q^{*}+2\right)}{\eta^{q^{*}+1}} \int_{0}^{\eta} \frac{(\eta-m)^{p^{*}+q^{*}-1}}{\Gamma\left(p^{*}+q^{*}\right)} v^{*}(m) \mathrm{d} m \\
& +\frac{\tau \Gamma\left(q^{*}+2\right)\left(\eta-\eta^{q^{*}+2}\right)}{\left(\eta^{q^{*}+2}-\eta^{q^{*}+1}\right)} \int_{0}^{1} \frac{(1-m)^{p^{*}+q^{*}-1}}{\Gamma\left(p^{*}+q^{*}\right)} v^{*}(m) \mathrm{d} m \\
& +\frac{\tau^{2} \Gamma\left(q^{*}+3\right)}{(\eta-1)} \int_{0}^{1} \frac{(1-m)^{p^{*}+q^{*}-1}}{\Gamma\left(p^{*}+q^{*}\right)} v^{*}(m) \mathrm{d} m \\
& -\frac{\tau^{2} \Gamma\left(q^{*}+3\right)}{\left(\eta^{q^{*}+2}-\eta^{q^{*}+1}\right)} \int_{0}^{\eta} \frac{(\eta-m)^{p^{*}+q^{*}-1}}{\Gamma\left(p^{*}+q^{*}\right)} v^{*}(m) \mathrm{d} m
\end{aligned}
$$

for each $\tau \in[0,1]$. Therefore $h^{*} \in A_{2} z^{*}$ and so $A_{2}$ has a closed graph. Hence $A_{2}$ is upper semi-continuous. Also, by hypothesis, the operator $A_{2}$ has compact values. Consequently, $A_{2}$ is a compact and upper semi-continuous operator.

Now, since by (N3)

$$
\begin{aligned}
M^{*}= & \left\|A_{2}(\mathcal{X})\right\|=\sup \left\{\left|A_{2} z\right|: z \in \mathcal{X}\right\} \\
= & {\left[\frac{1}{\Gamma\left(p^{*}+1\right)}+\frac{\Gamma\left(q^{*}+2\right) \eta^{p^{*}-1}}{\Gamma\left(p^{*}+q^{*}+1\right)}+\frac{\Gamma\left(q^{*}+2\right)\left|\eta-\eta^{q^{*}+2}\right|}{\left(\eta^{q^{*}+2}-\eta^{q^{*}+1}\right) \Gamma\left(p^{*}+q^{*}+1\right)}\right.} \\
& \left.+\frac{\Gamma\left(q^{*}+3\right)}{(\eta-1) \Gamma\left(p^{*}+q^{*}+1\right)}+\frac{\Gamma\left(q^{*}+3\right) \eta^{p^{*}+q^{*}}}{\left(\eta^{q^{*}+2}-\eta^{q^{*}+1}\right) \Gamma\left(p^{*}+q^{*}+1\right)}\right]\|s\|_{\mathcal{L}^{1}} \\
= & \Delta\|s\|_{\mathcal{L}^{1}} .
\end{aligned}
$$

So, letting $k^{*}=L^{*}$, we have $M^{*} k^{*}<\frac{1}{2}$. Therefore all assumptions of Theorem 2.3 hold for both $A_{1}$ and $A_{2}$. Then, one of conditions, condition (i) or condition (ii), holds.

We claim that condition (ii) is impossible. By Theorem 2.3 and by (N4), let $z$ be an arbitrary element of $\Sigma^{*}$ with $\|z\|=\tilde{\epsilon}$. Then $\mu z(\tau) \in A_{1} z(\tau) A_{2} z(\tau)$ for any $\mu>1$. Hence, we choose the function $v \in S_{\mathcal{H}, z}$ such that, for any $\mu>1$, we obtain

$$
\begin{aligned}
z(\tau)= & \frac{1}{\mu} \rho(\tau, z(\tau))\left[\int_{0}^{\tau} \frac{(\tau-m)^{p^{*}-1}}{\Gamma\left(p^{*}\right)} v(m) \mathrm{d} m-\frac{\tau \Gamma\left(q^{*}+2\right)}{\eta^{q^{*}+1}} \int_{0}^{\eta} \frac{(\eta-m)^{p^{*}+q^{*}-1}}{\Gamma\left(p^{*}+q^{*}\right)} v(m) \mathrm{d} m\right. \\
& +\frac{\tau \Gamma\left(q^{*}+2\right)\left(\eta-\eta^{q^{*}+2}\right)}{\left(\eta^{q^{*}+2}-\eta^{q^{*}+1}\right)} \int_{0}^{1} \frac{(1-m)^{p^{*}+q^{*}-1}}{\Gamma\left(p^{*}+q^{*}\right)} v(m) \mathrm{d} m \\
& +\frac{\tau^{2} \Gamma\left(q^{*}+3\right)}{(\eta-1)} \int_{0}^{1} \frac{(1-m)^{p^{*}+q^{*}-1}}{\Gamma\left(p^{*}+q^{*}\right)} v(m) \mathrm{d} m \\
& \left.-\frac{\tau^{2} \Gamma\left(q^{*}+3\right)}{\left(\eta^{q^{*}+2}-\eta^{q^{*}+1}\right)} \int_{0}^{\eta} \frac{(\eta-m)^{p^{*}+q^{*}-1}}{\Gamma\left(p^{*}+q^{*}\right)} v(m) \mathrm{d} m\right]
\end{aligned}
$$


for each $\tau \in[0,1]$. Thus, we get

$$
\begin{aligned}
& |z(\tau)|=\frac{1}{\mu}|\rho(\tau, z(\tau))|\left[\int_{0}^{\tau} \frac{(\tau-m)^{p^{*}-1}}{\Gamma\left(p^{*}\right)}|v(m)| \mathrm{d} m\right. \\
& +\frac{\tau \Gamma\left(q^{*}+2\right)}{\eta^{q^{*}+1}} \int_{0}^{\eta} \frac{(\eta-m)^{p^{*}+q^{*}-1}}{\Gamma\left(p^{*}+q^{*}\right)}|v(m)| \mathrm{d} m \\
& +\frac{\tau \Gamma\left(q^{*}+2\right)\left|\eta-\eta^{q^{*}+2}\right|}{\left(\eta^{q^{*}+2}-\eta^{q^{*}+1}\right)} \int_{0}^{1} \frac{(1-m)^{p^{*}+q^{*}-1}}{\Gamma\left(p^{*}+q^{*}\right)}|\nu(m)| \mathrm{d} m \\
& +\frac{\tau^{2} \Gamma\left(q^{*}+3\right)}{(\eta-1)} \int_{0}^{1} \frac{(1-m)^{p^{*}+q^{*}-1}}{\Gamma\left(p^{*}+q^{*}\right)}|v(m)| \mathrm{d} m \\
& \left.+\frac{\tau^{2} \Gamma\left(q^{*}+3\right)}{\left(\eta^{q^{*}+2}-\eta^{q^{*}+1}\right)} \int_{0}^{\eta} \frac{(\eta-m)^{p^{*}+q^{*}-1}}{\Gamma\left(p^{*}+q^{*}\right)}|v(m)| \mathrm{d} m\right] \\
& \leq[|\rho(\tau, z(\tau))-\rho(\tau, 0)|+|\rho(\tau, 0)|] \\
& \times\left[\int_{0}^{\tau} \frac{(\tau-m)^{p^{*}-1}}{\Gamma\left(p^{*}\right)}|v(m)| \mathrm{d} m+\frac{\tau \Gamma\left(q^{*}+2\right)}{\eta^{q^{*}+1}} \int_{0}^{\eta} \frac{(\eta-m)^{p^{*}+q^{*}-1}}{\Gamma\left(p^{*}+q^{*}\right)}|v(m)| \mathrm{d} m\right. \\
& +\frac{\tau \Gamma\left(q^{*}+2\right)\left|\eta-\eta^{q^{*}+2}\right|}{\left(\eta^{q^{*}+2}-\eta^{q^{*}+1}\right)} \int_{0}^{1} \frac{(1-m)^{p^{*}+q^{*}-1}}{\Gamma\left(p^{*}+q^{*}\right)}|\nu(m)| \mathrm{d} m \\
& +\frac{\tau^{2} \Gamma\left(q^{*}+3\right)}{(\eta-1)} \int_{0}^{1} \frac{(1-m)^{p^{*}+q^{*}-1}}{\Gamma\left(p^{*}+q^{*}\right)}|v(m)| \mathrm{d} m \\
& \left.+\frac{\tau^{2} \Gamma\left(q^{*}+3\right)}{\left(\eta^{q^{*}+2}-\eta^{q^{*}+1}\right)} \int_{0}^{\eta} \frac{(\eta-m)^{p^{*}+q^{*}-1}}{\Gamma\left(p^{*}+q^{*}\right)}|v(m)| \mathrm{d} m\right] \\
& \leq\left[L^{*}\|z\|+F^{*}\right]\left[\int_{0}^{\tau} \frac{(\tau-m)^{p^{*}-1}}{\Gamma\left(p^{*}\right)} s(m) \mathrm{d} m\right. \\
& +\frac{\tau \Gamma\left(q^{*}+2\right)}{\eta^{q^{*}+1}} \int_{0}^{\eta} \frac{(\eta-m)^{p^{*}+q^{*}-1}}{\Gamma\left(p^{*}+q^{*}\right)} s(m) \mathrm{d} m \\
& +\frac{\tau \Gamma\left(q^{*}+2\right)\left|\eta-\eta^{q^{*}+2}\right|}{\left(\eta^{q^{*}+2}-\eta^{q^{*}+1}\right)} \int_{0}^{1} \frac{(1-m)^{p^{*}+q^{*}-1}}{\Gamma\left(p^{*}+q^{*}\right)} s(m) \mathrm{d} m \\
& +\frac{\tau^{2} \Gamma\left(q^{*}+3\right)}{(\eta-1)} \int_{0}^{1} \frac{(1-m)^{p^{*}+q^{*}-1}}{\Gamma\left(p^{*}+q^{*}\right)} s(m) \mathrm{d} m \\
& \left.+\frac{\tau^{2} \Gamma\left(q^{*}+3\right)}{\left(\eta^{q^{*}+2}-\eta^{q^{*}+1}\right)} \int_{0}^{\eta} \frac{(\eta-m)^{p^{*}+q^{*}-1}}{\Gamma\left(p^{*}+q^{*}\right)} s(m) \mathrm{d} m\right] \\
& \leq\left[L^{*} \tilde{\epsilon}+F^{*}\right] \Delta\|s\|_{\mathcal{L}^{1}}
\end{aligned}
$$

for each $\tau \in[0,1]$. Hence, we obtain

$$
\tilde{\epsilon} \leq \frac{F^{*} \Delta\|s\|_{\mathcal{L}^{1}}}{1-L^{*} \Delta\|s\|_{\mathcal{L}^{1}}} .
$$

But by condition (10) we observe that condition (ii) of Theorem 2.3 is impossible. Therefore, the operator inclusion $z \in A_{1} z A_{2} z$ has a solution, and so the fractional hybrid differential inclusion (3) with three-point integral hybrid conditions (2) has at least one solution on $[0,1]$. 


\section{Examples}

In this section, to illustrate the importance and validity of our results, we present two examples.

Example 4.1 Our first example specifies the hybrid differential equation of the fractional order

$$
{ }^{c} \mathcal{D}_{0}^{\frac{9}{4}}\left(\frac{z(\tau)}{\frac{\tau|z(\tau)|^{2}}{1+|z(\tau)|^{2}}+4}\right)=\frac{\tau}{100} \sin z, \quad \tau \in[0,1]
$$

with three-point integral hybrid condition

$$
\begin{aligned}
& z(0)=0, \\
& \left.\left(\frac{z(\tau)}{\frac{\tau|z(\tau)|^{2}}{1+|z(\tau)|^{2}}+4}\right)\right|_{\tau=0}+\left.\mathcal{J}_{0}^{\frac{5}{4}}\left(\frac{z(\tau)}{\frac{\tau|z(\tau)|^{2}}{1+|z(\tau)|^{2}}+4}\right)\right|_{\tau=\frac{5}{4}}=0, \\
& \left.\left(\frac{z(\tau)}{\frac{\tau|z(\tau)|^{2}}{1+|z(\tau)|^{2}}+4}\right)\right|_{\tau=0}+\left.\mathcal{J}_{0}^{\frac{5}{4}}\left(\frac{z(\tau)}{\frac{\tau|z(\tau)|^{2}}{1+|z(\tau)|^{2}}+4}\right)\right|_{\tau=1}=0,
\end{aligned}
$$

where $p^{*}=\frac{9}{4}, q^{*}=\frac{5}{4}, \eta=\frac{5}{4}$. Consider the continuous function $\rho:[0,1] \times \mathbb{R} \rightarrow \mathbb{R} \backslash\{0\}$ by $\rho(\tau, z)=\frac{\tau|z|^{2}}{1+|z|_{2}}+4$ and the continuous function $\kappa:[0,1] \times \mathbb{R} \rightarrow \mathbb{R}^{+}$by $\kappa(\tau, z)=\frac{1}{100} \sin z$. It is evident that $L(\tau)=\tau$ and so $L^{*}=\sup _{\tau \in[0,1]}|L(\tau)|=1$. Also, we have $\sigma(\tau)=\frac{1}{100}$ and $\zeta(\|z\|)=$ 1. In this case, by given data, we get $\Delta=7.7283$. Therefore, we can choose $\epsilon>0.4187$, and consequently, we have $L^{*} \Delta M^{*} \zeta(\|z\|)=0.0772<1$. Now, Theorem 3.2 implies that the fractional hybrid differential equation (11) with three-point integral hybrid condition (12) has at least one solution on $[0,1]$.

Example 4.2 In the second example, we proceed to investigate the existence of solution for the fractional hybrid differential inclusion

$$
{ }^{c} \mathcal{D}_{0}^{\frac{5}{2}}\left(\frac{z(\tau)}{\tau^{2} \sin \frac{z(\tau)}{10}+3}\right) \in\left[\frac{|\cos z(\tau)|}{2(|\cos z(\tau)|+1)}, \frac{|\sin z(\tau)|^{2}}{3\left(1+|\sin z(\tau)|^{2}\right)}+\frac{2}{3}\right], \quad \tau \in[0,1]
$$

with three-point integral hybrid condition

$$
\begin{aligned}
& z(0)=0 \\
& \left.\left(\frac{z(\tau)}{\tau^{2} \sin \frac{z(\tau)}{10}+3}\right)\right|_{\tau=0}+\left.\mathcal{J}_{0}^{\frac{1}{2}}\left(\frac{z(\tau)}{\tau^{2} \sin \frac{z(\tau)}{10}+3}\right)\right|_{\tau=\frac{5}{4}}=0 \\
& \left.\left(\frac{z(\tau)}{\tau^{2} \sin \frac{z(\tau)}{10}+3}\right)\right|_{\tau=0}+\left.\mathcal{J}_{0}^{\frac{1}{2}}\left(\frac{z(\tau)}{\tau^{2} \sin \frac{z(\tau)}{10}+3}\right)\right|_{\tau=1}=0
\end{aligned}
$$

where $p^{*}=\frac{5}{2}, q^{*}=\frac{1}{2}, \eta=\frac{5}{4}$. Consider the continuous function $\rho:[0,1] \times \mathbb{R} \rightarrow \mathbb{R} \backslash\{0\}$ by $\rho(\tau, z)=\tau^{2} \sin \frac{z}{10}+3$ and the multifunction $\mathcal{H}:[0,1] \times \mathbb{R} \rightarrow \mathcal{P}(\mathbb{R})$ by

$$
\mathcal{H}(\tau, z)=\left[\frac{|\cos z|}{2(|\cos z|+1)}, \frac{|\sin z|^{2}}{3\left(1+|\sin z|^{2}\right)}+\frac{2}{3}\right]
$$


It is observed that $L(\tau)=\frac{\tau}{10}$ and so $L^{*}=\sup _{\tau \in[0,1]}|L(\tau)|=\frac{1}{10}$. Since, for each $h \in \mathcal{H}(\tau, z(\tau))$,

$$
|h| \leq \max \left(\frac{|\cos z|}{2(|\cos z|+1)}, \frac{|\sin z|^{2}}{3\left(1+|\sin z|^{2}\right)}+\frac{2}{3}\right) \leq 1, \quad z \in \mathbb{R}
$$

thus, we get

$$
\|\mathcal{H}(\tau, z)\|=\sup \{|v|: v \in \mathcal{H}(\tau, z)\} \leq 1
$$

Hence $s(\tau)=1$ for each $\tau \in[0,1]$ and so $\|s\|_{\mathcal{L}^{1}}=1$. By the above values, we get $\Delta=6.2371$. Therefore, we can find $\epsilon>0$ with $\tilde{\epsilon}>6.6298$. Finally, since $L^{*} \Delta\|s\|_{\mathcal{L}^{1}}=0.6237<\frac{1}{2}$, so by Theorem 3.4, the fractional hybrid differential inclusion (13) with three-point integral hybrid condition (14) has at least one solution on $[0,1]$.

\section{Conclusion}

By using Dhage's fixed point theorem, we provide some results about investigation of a hybrid type fractional differential equation and inclusion via some nonlocal three-point boundary value conditions. Finally, we provide two examples to illustrate our results.

\section{Acknowledgements}

The second and fourth authors were supported by Azarbaijan Shahid Madani University. The authors express their gratitude to dear unknown referees for their helpful suggestions which improved the final version of this paper.

\section{Funding}

Not available.

\section{Availability of data and materials}

Data sharing not applicable to this article as no datasets were generated or analyzed during the current study.

\section{Competing interests}

The authors declare that they have no competing interests.

\section{Authors' contributions}

The authors declare that the study was realized in collaboration with equal responsibility. All authors read and approved the final manuscript.

\section{Author details}

'Department of Mathematics, Cankaya University, Ankara, Turkey. ${ }^{2}$ Department of Mathematics, Azarbaijan Shahid Madani University, Tabriz, Iran. ${ }^{3}$ Young Researchers and Elite Club, Tabriz Branch, Islamic Azad University, Tabriz, Iran. ${ }^{4}$ Department of Medical Research, China Medical University Hospital, China Medical University, Taichung, Taiwan.

\section{Publisher's Note}

Springer Nature remains neutral with regard to jurisdictional claims in published maps and institutional affiliations.

Received: 5 August 2019 Accepted: 6 November 2019 Published online: 14 November 2019

\section{References}

1. Agarwal, R.P., Ntouyas, S.K., Ahmad, B., Alhothuali, M.: Existence of solutions for integro-differential equations of fractional order with nonlocal three-point fractional boundary conditions. Adv. Differ. Equ. 2013, Article ID 128 (2013)

2. Ahmad, B., Alsaedi, A., Ntouyas, S.K., Tariboon, J.: Hadamard-Type Fractional Differential Equations, Inclusions and Inequalities. Springer, Berlin (2017)

3. Ahmad, B., Etemad, S., Ettefagh, M., Rezapour, Sh.: On the existence of solutions for fractional q-difference inclusions with q-antiperiodic boundary conditions. Bull. Math. Soc. Sci. Math. Roum. 59(107)(2), 119-134 (2016)

4. Ahmad, B., Ntouyas, S.K.: Initial value problems for hybrid Hadamard fractional differential equation. Electron. J. Differ. Equ. 2014, Article ID 161 (2014)

5. Ahmad, B., Ntouyas, S.K., Alsaedi, A.: Existence results for a system of coupled hybrid fractional differential equations. Sci. World J. 2014, Article ID 426438 (2014)

6. Ahmad, B., Ntouyas, S.K., Alsedi, A.: On fractional differential inclusions with anti-periodic type integral boundary conditions. Bound. Value Probl. 2013, 82 (2013)

7. Ahmad, B., Ntouyas, S.K., Tariboon, J.: A nonlocal hybrid boundary value problem of Caputo fractional integro-differential equations. Acta Math. Sci. 36(6), 1631-1640 (2016) 
8. Aubin, J., Cellna, A.: Differential Inclusions: Set-Valued Maps and Viability Theory. Springer, Berlin (1984)

9. Aydogan, S.M., Baleanu, D., Mousalou, A., Rezapour, Sh.: On approximate solutions for two higher-order Caputo-Fabrizio fractional integro-differential equations. Adv. Differ. Equ. 2017, Article ID 251 (2017)

10. Aydogan, S.M., Baleanu, D., Mousalou, A., Rezapour, Sh.: On high order fractional integro-differential equations including the Caputo-Fabrizio derivative. Bound. Value Probl. 2018, 90 (2018)

11. Baleanu, D., Ghafarnezhad, Kh., Rezapour, Sh.: On a three steps crisis integro-differential equation. Adv. Differ. Equ. 2019, Article ID 153 (2019)

12. Baleanu, D., Ghafarnezhad, Kh., Rezapour, Sh., Shabibi, M.: On the existence of solutions of a three steps crisis integro-differential equation. Adv. Differ. Equ. 2018, Article ID 135 (2018)

13. Baleanu, D., Hedayati, V., Rezapour, Sh., Al-Qurashi, M.M.: On two fractional differential inclusions. SpringerPlus 5 Article ID 882 (2016)

14. Baleanu, D., Khan, H., Jafari, H., Khan, R.A., Alipour, M.: On existence results for solutions of a coupled system of hybrid boundary value problems with hybrid conditions. Adv. Differ. Equ. 2015, Article ID 318 (2015)

15. Baleanu, D., Mohammadi, H., Rezapour, Sh.: On a nonlinear fractional differential equation on partially ordered metric spaces. Adv. Differ. Equ. 2013, Article ID 83 (2013)

16. Baleanu, D., Mohammadi, H., Rezapour, Sh.: Some existence results on nonlinear fractional differential equations. Philos. Trans. R. Soc. Lond. A 371, 20120144 (2013)

17. Baleanu, D., Mohammadi, H., Rezapour, Sh.: The existence of solutions for a nonlinear mixed problem of singular fractional differential equations. Adv. Differ. Equ. 2013, Article ID 359 (2013)

18. Baleanu, D., Mousalou, A., Rezapour, Sh.: A new method for investigating approximate solutions of some fractional integro-differential equations involving the Caputo-Fabrizio derivative. Adv. Differ. Equ. 2017, Article ID 51 (2017)

19. Baleanu, D., Mousalou, A., Rezapour, Sh.: On the existence of solutions for some infinite coefficient-symmetric Caputo-Fabrizio fractional integro-differential equations. Bound. Value Probl. 2017, 145 (2017)

20. Baleanu, D., Mousalou, A., Rezapour, Sh.: The extended fractional Caputo-Fabrizio derivative of order $0 \leq \sigma<1$ on $C_{\mathbb{R}}[0,1]$ and the existence of solutions for two higher-order series-type differential equations. Adv. Differ. Equ. 2018, Article ID 255 (2018)

21. Baleanu, D., Rezapour, Sh., Etemad, S., Alsaedi, A.: On a time-fractional integro-differential equation via three-point boundary value conditions. Math. Probl. Eng. 2015, Article ID 785738 (2015)

22. Bragdi, M., Debbouche, A., Baleanu, D.: Existence of solutions for fractional differential inclusions with separated boundary conditions in Banach space. Adv. Math. Phys. 2013, Article ID 426061 (2013)

23. Deimling, K:: Multi-valued Differential Equations. de Gruyter, Berlin (1992)

24. Dhage, B.C.: Existence results for neutral functional differential inclusions in Banach algebras. Nonlinear Anal. 64 1290-1306 (2006)

25. Dhage, B.C.: Nonlinear functional boundary value problems in Banach algebras involving Carathéodories. Kyungpook Math. J. 46, 527-541 (2006)

26. Dhage, B.C., Lakshmikantham, V.: Basic results on hybrid differential equation. Nonlinear Anal. Hybrid Syst. 4, 414-424 (2010)

27. Herzallah, M.A.E., Baleanu, D.: On fractional order hybrid differential equations. Abstr. Appl. Anal. 2014, Article ID $389386(2014)$

28. Hristova, S., Tunc, C.: Stability of nonlinear Volterra integro-differential equations with Caputo fractional derivative and bounded delays. Electron. J. Differ. Equ. 2019, Article ID 30 (2019)

29. Khan, H., Tunc, C., Chen, W., Khan, A.: Existence theorems and Hyers-Ulam stability for a class of hybrid fractional differential equations with P-Laplacian operator. J. Appl. Anal. Comput. 8(4), 1211-1226 (2018)

30. Lasota, A., Opial, Z: An application of the Kakutani-Ky Fan theorem in the theory of ordinary differential equations. Bull. Acad. Pol. Sci., Sér. Sci. Math. Astron. Phys. 13, 781-786 (1965)

31. Mahmudov, N., Matar, M.: Existence of mild solutions for hybrid differential equations with arbitrary fractional order TWMS J. Pure Appl. Math. 8(2), 160-169 (2017)

32. Miller, S., Ross, B.: An Introduction to the Fractional Calculus and Fractional Differential Equations. Wiley, New York (1993)

33. Ntouyas, S.K., Etemad, S.: On the existence of solutions for fractional differential inclusions with sum and integral boundary conditions. Appl. Math. Comput. 266, 235-243 (2015)

34. Ouahab, A.: Some results for fractional boundary value problem of differential inclusion. Nonlinear Anal. 69 , 3877-3896 (2008)

35. Phung, P.D., Truong, L.X.: On a fractional differential inclusion with integral boundary conditions in Banach space. Fract. Calc. Appl. Anal. 16(3), 538-558 (2013)

36. Podlubny, I.: Fractional Differential Equations. Academic Press, San Diego (1999)

37. Samei, M.E., Hedayati, V., Rezapour, Sh.: Existence results for a fractional hybrid differential inclusion with Caputo-Hadamard type fractional derivative. Adv. Differ. Equ. 2019, Article ID 163 (2019)

38. Samko, G., Kilbas, A., Marichev, O.: Fractional Integrals and Derivatives: Theory and Applications. Gordon \& Breach, New York (1993)

39. Sun, S., Zhao, Y., Han, Z., Li, Y.: The existence of solutions for boundary value problem of fractional hybrid differential equations. Commun. Nonlinear Sci. Numer. Simul. 17, 4961-4967 (2012)

40. Zhao, Y., Sun, S., Han, Z., Li, Q.: Theory of fractional hybrid differential equations. Comput. Math. Appl. 62, 1312-1324 (2011) 